\title{
Natural Cybernetics of Time, or about the Half of any Whole
}

\author{
Vasil Penchev, vasildinev@gmail.com \\ Bulgarian Academy of Sciences: Institute of Philosophy and Sociology: \\ Dept. of Logic and Philosophy of Science
}

\begin{abstract}
Norbert Wiener's idea of "cybernetics" is linked to temporality as in a physical as in a philosophical sense. "Time orders" can be the slogan of that natural cybernetics of time: time orders by itself in its "screen" in virtue of being a well-ordering valid until the present moment and dividing any totality into two parts: the well-ordered of the past and the yet unordered of the future therefore sharing the common boundary of the present between them when the ordering is taking place by choices. Thus, the quantity of information defined by units of choices, whether bits or qubits, describes that process of ordering happening in the present moment. The totality (which can be considered also as a particular or "regional" totality) turns out to be divided into two parts: the internality of the past and the externality of the future by the course of time, but identifiable to each other in virtue of scientific transcendentalism (e.g. mathematical, physical, and historical transcendentalism). A properly mathematical approach to the "totality and time" is introduced by the abstract concept of "evolutionary tree" (i.e. regardless of the specific nature of that to which refers: such as biological evolution, Feynman trajectories, social and historical development, etc.), Then, the other half of the future can be represented as a deformed mirror image of the evolutionary tree taken place already in the past: therefore the past and future part are seen to be unifiable as a mirrorly doubled evolutionary tree and thus representable as generalized Feynman trajectories. The formalism of the separable complex Hilbert space (respectively, the qubit Hilbert space) applied and further elaborated in quantum mechanics in order to uniform temporal and reversible, discrete and continuous processes is relevant. Then, the past and future parts of the evolutionary tree would constitute a wave function (or even only a single qubit once the concept of actual infinity be involved in real processes). Each of both parts of it, i.e. either the future evolutionary tree or its deformed mirror image, would represent a "half of the whole". The two halves can be considered as the two disjunctive states of any bit as two fundamentally inseparable (in virtue of quantum correlation) "halves" of any qubit. A few important corollaries exemplify that natural cybernetics of time.
\end{abstract}

Keywords: cybernetics, evolutionary tree, Feynman pathway, quantum correlation, quantum holism, quantum information, qubit, the totality, time 


\section{INSTEAD OF INTRODUCTION: THE PROBLEM ABOUT THE "HALF OF THE TOTALITY"}

The problem about the "half of the totality" consists in the ostensible contradiction of the following two statements:

1. The half of the totality is a true part of the totality.

2. The half of the totality coincides with the totality since the half of the totality satisfies the definition of the totality itself.

Anyway, the contradiction is ostensible as both can be involved in an alternative definition of the totality following the paradigm of "actual infinity" in set theory. That is: as an actually infinite set contents at least a true subset (e.g. such as one consisting of the half number of its elements) so that there exists a bijection between both, as the totality contents at least a true part of it (e.g. such one defined as the half of the totality) so there exists an identity between both.

The term the "half of the totality" is chosen initially for its intuitive sense to juxtapose the latter "identity" to the former "bijection". Indeed, if the "half of an actual infinite set" can be mapped one-to-one into the same (or any other, in fact) actual infinite set, the complement (to the actual infinite set) of the "half of an actual infinite set" can be mapped into it unambiguously as well, and thus, there exists another bijection of the half and "other half" (i.e. the complement of the half) of any actually infinite set therefore justifying the intuitive sense of the term "half".

Even more, the term "half" is especially, much more fruitful in relation to "the totality" than to "infinite set" because of the following consideration:

The totality can be defined for the objectivity of scientific (i.e. falsifiable) transcendentalism as "doubling by itself" in virtue of the definitive bijection of its "externality" and its "internality" and the substitution of the internality by the elements of the set of the bijection at issue. All those "elements of the bijection" can be identified as the "internality" (though not less as the "externality") of the totality therefore able to fetch any external element from its internal counterpart: the bijection is just that fetching.

The analogy (but isomorphism, in fact, after a more rigorous enough consideration) of the totality and infinite set can be continued in relation to distinguishing finite and infinite subsets of an infinite set according to the absence/ existence of that bijection as above correspondingly:

One can define a whole (or "wholeness") as the counterpart of an infinite subset in the transcendental theory (thus falsifiable) of the totality by the existence of that identity to the totality as above. Speaking loosely, any whole (wholeness) is a "particular totality" being a true part of the totality but nonetheless sharing the definitive property of the totality: to contain its externality in itself by itself.

Thus and particularly, the mind - body problem of Descartes dualism or the analogical relation "subject - object" in the classical German philosophy can be resolved both successfully and rather trivially ("definitively"): Kant's "Copernican revolution" of transcendentalism is historically first.

Furthermore, one can define those parts of the totality which are not whole by the absence of any identity between each of them and the totality and thus corresponding to "finite subsets" of any infinite set.

By set theory, mathematics resolved rather later the same essential problem underlying Kant's transcendentalism and the further development of Western philosophy. However, their particular solutions managed to discover also: physics by thermodynamics and then, by quantum mechanics, biology by evolutionary theory and ecology, psychology by the Gestalt theory, etc. 
All of them share Wittgensteinian "family resemblance", but their Wittgenstenian "ladder" will be removed achieving their counterparts in philosophy and mathematics therefore building a uniform, formal and mathematical theory of the totality and wholeness. It turns out to be linked naturally and directly to Wiener's idea of cybernetics by the "natural cybernetics of time" sketchable by the present research.

Wiener (1948) described the "new science of cybernetics" as studying general laws of control in systems such as technical or biological (generalizable easily to any other embodiment of the concept of system ${ }^{1}$ ) by the transfer and processing of information ${ }^{2}$. One can demonstrate that the above examples of the general theory of the totality and whole can be linked to the general cybernetic theory of systems supposedly extending the latter to any system featuring by temporal processes what all natural physical systems or all social and historical systems are.

That generalization is what is meant by "natural cybernetics of time".

\section{THE PROBLEM ABOUT THE HALF OF A WHOLE IN SCIENCE: A FEW EXEMPLIFICATIONS}

At least a few sciences have met the problem, outlined in the previous section, in their own terms and have been forced to find specific relevant solutions. One of the objectivity of the natural cybernetics of time is to suggest a general mathematical theory of them, but they should be enumerated and featured briefly before that:

Thermodynamics existing in a few modifications such as phenomenological (Carnot's), statistic (Boltzmann's, Gibbs's, and Einstein's), etc. (which are out of the present scope) is presumably the first experimental and mathematized science started to study a particular form of wholeness: the physical (more precisely, mechanical) one.

Phenomenological thermodynamics studies a few physical quantities and their relations attributable to all bodies in our usual macroscopic experience. However, the fact that they refer only to a special whole (statistical ensemble) remained hidden and thus implicit until the discovery of statistic approach to phenomenological thermodynamics and consisting on the postulate that any thermodynamic state can be decomposed into different sub-states interpreted differently in the enumerated three versions: sub-states associable with the mechanical states of "atoms", "molecules", etc., a huge number of which constitutes a statistical ensembles in Boltzmann's; sub-states associable with all possible thermodynamic states of the investigated thermodynamic whole in Gibbs's; or sub-states associable with all possible thermodynamic (or even mechanics) interaction of the whole with other macroscopic entities in Einstein's.

One can consider those three statistic approaches from the viewpoint of the metaphysical problem about the "half of a whole" as follows:

Boltzmann's theory means the relations of a whole being wholeness in the meaning of a particular totality as above and its parts such as atoms, molecules, etc. which are only finite parts, but each of them cannot be accepted to be as a thermodynamic whole in that kind of research (though they are granted to be "inseparable" mechanical elements). Then, Boltzmann's approach can be illustrated withal very instructively even as purely mathematical meaning conclusions of the previous section:

\footnotetext{
${ }^{1}$ Wiener considered social, philosophical, and even theological sequences of cybernetics in two other papers $(1950,1964)$.

${ }^{2}$ Shannon (1948) introduced the quantity of information in the cited paper of the same year.
} 
One can build consistently a properly thermodynamic, Boltzmann theory of "mechanically moving" natural numbers in the meaning of Peano arithmetic (or more complex arithmetical structures if need be) therefore constituting a whole such as a transfinite natural number in "Hilbert arithmetic" and associable unambiguously with a "wave function" (as e.g. in: Penchev 2020 July 20) in turn interpretable as the characteristic function of the probability distribution of an "ensemble of moving natural numbers". However, that consideration would need rather Gibbs's approach as a mediator between Boltzmann's and that of quantum mechanics (once "wave function" has been involved) as in the following paragraphs of this section.

Gibbs's theory excludes Boltzmann's thermodynamic "elements" (i.e. atoms, molecules, etc.) substituting them by all possible states of the investigated macroscopic and thermodynamic entity therefore observable in possible experiments whether real or thought (i.e. Einstein's "Gedankenexperimenten"). Anyway his idea is not less revolutionary than Boltzmann's postulated experimentally inaccessible (at least then) elements. Indeed, Gibbs described a whole by the statistical ensemble of all its possible states. Implicitly, this is an extraordinary and very radical idea: the actual reality (such as that of an empirical entity meant by thermodynamics) to be described as the unity of its complete virtual versions (i.e. "virtuality") or, therefore to be decomposable to them exhaustively and thoroughly, an idea which quantum mechanics will transfer into mechanics in order to be able to uniform the quantum (discrete) changes forced by the Planck constant, on the one hand, and their readings by the apparatus described by the smooth (continuous) classical mechanics, on the other hand.

The corresponding "Gibbs arithmetic of moving natural numbers" would introduce explicitly the unambiguous identification of just one finite natural number of the one twin Peano arithmetic and just one transfinite natural number (a wave function) of the other twin Peano arithmetic and idempotently complementary to the former. That "Gibbs arithmetic" is to interpret the former natural number as a real state absolutely describable by the latter "moving natural number" (i.e. "transfinite natural number) as the collection of all possible states of the real state meant by the former.

Those exemplifications whether by "Boltzmann arithmetic" or "Gibbs arithmetic" (both being in the framework of Hilbert arithmetic) need an extraordinary kind of "fundamental ontology", or "first philosophy", namely quantum and neo-Pythagorean, thus inconsistent, incompatible, and even incommensurable with any representative of all the class of admissible contemporary Western ontologies originating from, realizing, or relevant to the "abyss" of Cartesian dualism: its "apple of sin" has to have not been eaten, even not been offered. One must return to the age before Descartes and his choice predetermined Western philosophy in order to follow a branch of intellectual history counterfactual to ours.

The main difference would be that there would be a smooth transition between mathematical structures (i.e. Pythagorean "Numbers") and the entities of Modern physics rather than the unsurmountable abyss of dualism between them. Unfortunately, one need overcome all the Gestalt of contemporary cognition, its historically welded episteme, an intellectual feat in order for a new worldview and perspective of development to be unveiled.

That new Gestalt will be necessary further for the natural cybernetics of time to be constructible. Apropos, Wiener's original cybernetics contents the same idea implicitly searching for that Gestalt in the framework of which technical and biological systems might be considered uniformly. Analogically, the present research is directed to a general informational approach to all 
temporal systems (what all natural ones are) to reveal the ways by which they control themselves by themselves, conserving their wholeness.

After Boltzmann's thermodynamics and Gibbs's thermodynamics, Einstein's one is to be seen by the problem about the "half of wholeness". His innovation can be interpreted from that viewpoint as adding a relevant thermodynamic (and even mechanical) environment to the thermodynamic whole once it has been considered in Gibbs's manner (or in the correspondence of Gibbs's manner to Boltzmann's one). Then, one need investigate both linked and correlative copies of wholeness: those of the entity and its environment both representable by Gibbs's method, therefore closer and closer approaching the way for quantum mechanics to be established.

The corresponding "Einstein's arithmetic of moving natural numbers", accordingly, would involve explicitly both complimentary twins of Hilbert arithmetic: the one for the thermodynamic entity at issue, the other one for its environment, moreover both exchangeable idempotently. Even more, the two twin arithmetical variables can be independent of each other therefore implying a kind of "arithmetical interaction" (i.e. an analogue of physical interactions) being a functions of those two independent variables, each of which belonging to the one of the two complimentary copies of Peano arithmetic.

The step of quantum mechanics forced by the fundamental Planck constant can be interpreted directly in terms of Hilbert arithmetic. Unlike the so-called Einstein arithmetic, which differs the "arithmetical interactions" therefore introducing the simultaneous consideration of natural numbers of the two complimentary twins, Hilbert arithmetic (being furthermore isomorphic to the qubit, or to the separable complex Hilbert space of quantum mechanics) considers rather the class of equivalence of all those "arithmetical interactions" complementarity in virtue of the implicit equivalence of the two twins, withal, Hilbert arithmetic expresses that implicit equivalence explicitly by adding still, one "third" Peano arithmetic,

${ }^{3}$ That class of equivalence underlies the contemporary quantum mechanics for it wac constituted by merging Heisenberg's matrix mechanics and Schrödinger's wave mechanics therefore needing the property of unitarity to be able to do this (Penchev 2020 June 21). Its fundamental base can be found in set theory: in the well-known and almost trivial equivalence of the well-ordering theorem and the axiom of choice. An even deeper foundation representc "transcendental invariance" (Penchev 2020 August 31) inferable from ,scientific transcendentalism“meaning a philosophical equivalence or link of well-ordering (time) and choice (information). 
however, interpreted "non-standardly" unlike the first two ones being complementary to each other, but both standard".

The unity of the wholeness (by the one twin) and its environment (by the other twin) is supplied by two alternative ways in "Einstein arithmetic" versus Hilbert arithmetic:

1. "Einstein arithmetic": both twins are linked to each other on their own level therefore suspending "complementarity" featuring quantum mechanics (at least in Niels Bohr's interpretation).

2. Hilbert arithmetic: both twins are linked to each other externally, i.e. by an external framework (such as the "third" and nonstandard Peano arithmetic) thus being situated on the meta-level to that of the twins therefore keeping the "complementarity" of quantum mechanics as far as only the one twin is available actually in the common shared framework.

"Einstein arithmetic" and Hilbert arithmetic are complementary rather than inconsistent to each other: both express the same in different (complimentary) ways. That "same" is the unity of the twins represented on their own level by the former (by the "arithmetic interaction") or the meta-level by the latter (by the third, nonstandard Peano arithmetic). The two descriptions can be equated $^{6}$ to each other in virtue of expressing the same: the "peaceful" (i.e. consistent) coexistence of the twins.

As a conclusion, Hilbert arithmetic offers a framework general enough to be able to uniform all the particular phenomena of the problem about the "half of wholeness" in the three versions of

\footnotetext{
${ }^{4}$ The "nonstandard interpretation of Peano arithmetic" is meant as comprising both complimentary twins, i.e. extended between the least finite natural number " 1 " and the greatest transfinite natural number " $\omega$ " (where " $\omega$ " notates as usual the least ordinal among those of all actual infinite sets, speaking loosely). The function successor is defined "non-standardly" as well: namely as " $n=$ "; so that " 1 " $\stackrel{\text { def }}{=} 1=; 2 \stackrel{\text { def }}{=} 1=1=; 3 \stackrel{\text { def }}{=} 1=1=1=$, etc. Thus, the nonstandard interpretation of Peano arithmetic is "nonstandard" in a sense opposite to that of any "nonstandard interpretation in set theory" (which means a countable model for any set supplied by any structure in virtue of the Löwenheim - Skolem theorem). Indeed, that direction of interpretation is reversed as to the nonstandard interpretation of Peano arithmetic since a model of an arbitrary power is constructed for an enumerable set in general. Particularly, that nonstandard interpretation can be considered as cyclic because " $1=\omega$ " is true as to it. Figuratively, each of both complementary twins can be obtained after "cutting" the cyclic structure as a necessary condition for it to be well-ordered. A much more detailed consideration and inference is contained in: Penchev 2020 July 20).

${ }^{5}$ Both twin complimentary standard interpretations of Peano arithmetic differ from each other (though in an idempotent way) by the following two definitive properties: (1) The one starts from " 1 " with (2) the function successor " $n+1$ ", but (1) the other one begins from " $\omega$ " with (2) the function successor "n- 1 " (more in: Penchev 2020 July 20).

${ }^{6}$ The sense of that equating would be clearer after possibly introducing the relevant correlate of an eventual "Einstein arithmetic" just as the qubit Hilbert space is the complimentary correlate of Hilbert arithmetic: it would turn out to be the pseudo-Riemannian space of general relativity where any class of equivalence, what any wave function represents, is "broken" into elements different from each other by a special tensor "quantity of curvature" or its field defined in any space-time point. So, the essence is that a class of equivalence is equated to all the elements of the same class and relying, in the final analysis, on the equivalence of the well-ordering "theorem" and the axiom of choice (valid at least in set theory). Particularly, that "Einstein arithmetic" can be expressed in terms of Hilbert arithmetic, and the correlative image of which is a theory of entanglement as equivalent to gravitation (as it is represented in general relativity; more in: Penchev 2020 August 31).
} 
statistical thermodynamics, on the one hand, and quantum mechanics ${ }^{7}$, on the other hand. It can be reformulated by its solution in terms of Hilbert arithmetic as follows:

Any bit of (classical) information and constituted by the three "number-sake" (i.e. name-sake) natural numbers resolves the problem by implicitly involving a level (for the two halves) and a meta-level (for their wholeness) if the natural numbers are finite. Not only analogically, but equivalently, any qubit of (quantum) information and constituted in the same way, but for "transfinite natural numbers", resolves the problem by implicitly involving two entangled (in general) qubits (for the two halves) and still one, third qubit for their system (entangled in general).

What is forthcoming is the utilization of Hilbert arithmetic to be represented by "abstract evolutionary tree" such as that in theory of biological evolution, or linguistic evolution, or social and political evolution, or any other temporal evolution in the final analysis. This is intended as a "key" for mathematicizing the corresponding scientific areas for Hilbert arithmetic (respectively the qubit Hilbert space) is mathematical enough to be able to "unlock" them.

\section{THE CONCEPT OF ABSTRACT EVOLUTIONARY TREE}

The usual "tree" of biological evolution can suggest a visualization for the idea of an abstract "evolutionary tree". Another one is that of linguistic evolution or the scheme of any evolution happening in time.

It can be described as a graph bifurcating more and more into more and more branches (likenable to a "tree") following a relevant scale of time, but specific as to any certain process. All those branches are real (actual) and resulting into corresponding entities clearly distinguishable from each other.

Any branch starts by a bifurcation from the parent branch and consists in following an alternative after an "evolutionary event" admitting different "choices" (with or without quotation marks). Imaginary or discursive (narrative) branches, i.e. counterfactual, can complement the tree at issue even transforming it into a dense, continuous or smooth medium liken it as a space-time physical field.

Then, the differences of abstract evolutionary tree to quantum field (for example, visualizable by Feynman pathways, partly similar to a "tree") are only a few and only they restrict or feature the utilization of the separable complex Hilbert space of quantum mechanics for abstract evolutionary three. Those are:

1. Quantum field is dense, continuous or smooth, defined in any point of space-time, and the abstract evolutionary field is not, but consists of separate branches discretely divided from each

\footnotetext{
7 Indeed, quantum mechanics itself can be interpreted as a (statistical) thermodynamic theory of mechanical motion forced by the Planck constant in order to make clear its relation to the enumerated three versions of statistical thermodynamics. Here is how one has utilized the Gibbs paradigm, for example. Due to the Planck constant, any quantum change can be interpreted as the change of a thermodynamic whole representable exhaustively by all its possible states as to what its wave function can be seen. Once the Gibbs approach is demonstrated as possible, the other two ones follow after simply translating their languages correspondingly in that of quantum mechanics. However, still one and very essential "peculiarity" of quantum mechanics exists: in comparison with statistical thermodynamics (non-quantum) and also due to the Planck constant for it forbids to be considered different states "within a single quantum" therefore forcing they to be identified definitively only as a class. In fact, this is a well-known corollary after the famous theorems about the absence of hidden variables in quantum mechanics (Neumann 1932; Kochen, Specker 1967).
} 
other. Anyway, the latter can be complemented to the former "counterfactually", i.e. by a dense network of counterfactual branches.

2. Quantum field is virtual or probabilistic, but abstract evolutionary field is actual, and any branch in it happens really, in parallel to all others. Anyway, quantum fields can be interpreted also as actual if one does this in Everett's manner. Indeed, the transition between any two parallel "worlds" (among the "many ones") is impossible (or "forbidden") just as that between two branches of the abstract evolutionary tree. Furthermore, the irreversibility of the time arrow is conserved in both unlike the reversible coherent state of a relevant collection of trajectories, each of which supplied by a certain probability to happen really (i.e. as measured).

3. Quantum field is not "tree-like", but rather similar to a "bulb" or a "globe", the two poles of which are the initial state and final state of quantum change correspondingly. Then, two abstract evolutionary trees arbitrary in general and following opposite time arrows can be unified into a single quantum field; or vice versa: the latter is decomposable to two copies of the former.

Just that third difference ${ }^{8}$ can help to resolve the problem of the half of a whole in a way linkable to abstract evolutionary trees. The "second tree" (complementing the first one to quantum field) can be interpreted in a few ways, each of which is relevant to different scientific areas:

1. As the same tree (as the first one), but in the opposite direction of time. The representation by quantum field adds it as a mirror image to the "end" of the first (real) tree.

2. As an arbitrary tree, but in the opposite direction of time, and obeys the condition for the first tree to continue into the second one smoothly or at least continuously therefore defining a class of equivalence of states of quantum field by sharing both trees.

3. Both 1 and 2 can be doubled by still one (in fact, two) representations of two complementary trees in the same direction of time (for each of both).

Thus, information (or quantum information if need be) is a quantity relevant to abstract evolutionary trees. Any state of an abstract evolutionary tree whether single or doubled defines a class of equivalence of wave functions sharing the state at issue.

Anyway, the essential difference mentioned above is kept: the Feynman trajectories of wave function are virtual, but the branches of abstract evolutionary tree are real (actual), on the one hand, and on the other hand, the wave function contains additional information consisting in the quantity of probability (whether constant or variable) for each branch to happen after measurement in a certain moment of time. The two mismatches are to be overcome one by one:

1. One can add weighting factors (eventually normed to their total sum) in a way corresponding to the interpretation of corresponding evolutionary tree in each case of its utilization. For example, the number of individuals of a population of evolutionary species in its dynamics or the number of people speaking a certain language in its dynamics, etc. Then, the evolution from the initial state (such as a proto-species or a proto-language) would take place simultaneously and follow all evolutionary branches just as if a quantum "semi-leap" between two discretely separate states of a change.

2. In a close link to 1 , one can distinguish the real trajectory from the virtual trajectory according to the opposition of irreversible - reversible time correspondingly. Indeed, reversible time means the complement of the time arrow by means of its opposite direction, after which the quantity of time needing well-ordering turns out to be undefinable since the coherent state is not

\footnotetext{
${ }^{8}$ The first and second ones can be considered rather as "surmountable obstacles".
} 
well-ordered fundamentally and definitively. Thus, irreversible time can be acquired from coherent state only by taking away rather by adding'.

In other words, one can obtain the "real picture" from the virtual one removing the second, complimentary (and as if qualifiable as "redundant") twin of the real picture, but "backward" in time, and implicitly available in the corresponding coherent state ("virtual picture") together with that "forward" in time (i.e. "real picture"). Furthermore and speaking figuratively, the twins can be as "monozygotic" as "dizygotic", i.e. as identical as non-identical. The difference between them is zero in the former case, but a relatively arbitrary quantity in the latter. One can consider also the class of equivalence of all possible dizygotic twins of the same parents in a certain moment and identify that class any of both relevant pairs of monozygotic twins.

The last sentence can be interpreted as a metaphor about the relation of general relativity and quantum mechanics: the former means "dizygotic twins" (the one is a contravariant tensor, the other one is a covariant tensor) therefore admitting the quantity of their mismatch variable in general and linkable to gravitational interaction. On the contrary, the description of quantum mechanics would exclude the option of their mismatch considering all twins as monozygotic or at least as representable well by the case of monozygotic twins ${ }^{10}$. However, even quantum mechanics contains the option for "dizygotic twins" to be discussed in terms of its language: those are all phenomena of entanglement mean in the theory of quantum information ${ }^{11}$.

If the lesson taught by the so-called quantum gravity about the cases of "monozygotic and dizygotic twins" (only in the sense of the above metaphor) in mathematical terms is mastered, it can be reinterpreted immediately as to abstract evolutionary tree and to the consideration a few paragraphs above notated as " 2 ".

Once the theory of quantum information has been involved as relevant to abstract evolutionary tree, it turns out to be doubled (or more precisely, "doublable": i.e. capable of being doubled) by means of two complementary ways: either as two identical (monozygotic') abstract evolutionary trees or as two non-identical (dizygotic) ones; withal the former means the class of all latter ones.

So one needs a relevant interpretation of the "second twin" being non-identical to the first one possessing a clear interpretation as granted in any certain case where an abstract evolutionary tree is utilized.

\footnotetext{
${ }^{9}$ A few philosophical and physical conclusions inferable from that observation are discussed in a previous paper (Penchev 2019).

${ }^{10}$ In fact, the equivalent description in terms of a single quantum system (i.e. by the case of "monozygotic twins") is possible always; and then: "no room for gravity" since it (at least in the framework of general relativity) describes the case of "dizygotic twins". However, one may state not less that the case of "dizygotic twins" is universal, and that of "monozygotic twins" is a particular case in the framework of the former; then, the phenomena of entanglement and their theory of quantum information would be to be considered as general accordingly, and all studied by the "classical" quantum mechanics (meaning one single quantum mechanics, however, equivalent to many ones, but necessarily non-entangled) until now is the particular case relevant only to matter and energy which are not "dark". That extraordinary epistemological relation is the subject of discussion in: Penchev 2020 August 30.

${ }^{11}$ The necessary existence of entanglement and all phenomena implied by it (among the most important ones is "dark field" unifying "dark energy" and "dark matter") can be deduced even still from the postulate of

the totality by means of "physical and mathematical transcendentalism" (in detail in: Penchev 2020 August 31) via the "conservation of quantum information" (in detail in: Penchev 2020 August 17).
} 
Here are a few exemplifications of those interpretations of the "second twin" being non-identical. For example, as to the case of biological evolution: environment suggests an alternative potential evolutionary tree of ecological niches, some of which are not dwelt yet, others are occupied recently, and third ones are mastered a long time ago. Then, a kind of interaction can be defined between that "potential evolutionary tree", on the one hand, and the real, usual and actual evolutionary tree of species at the same historical moment. That interaction would be the "mover" or "propulsion" of evolution conditioning changes and thus, future developments of the actual evolutionary tree.

Anyway, one can suggest at least still one version for interpreting that "second twin" of evolutionary tree in biology originating from the equivalent biochemical DNA (or RNA) image of

the actual evolutionary tree in a certain historical moment. One can imagine and then calculate counterfactual, but stable states (i.e. possible mutations) as that potential evolutionary tree acting on the actual by "force of attraction".

As a particular DNA case, one can consider the process for an ovum to be fertilized. A necessary condition for that to happen is both branches of DNA (i.e. the paternal "half" and maternal "half") to be close enough to each other, almost identical such as those of two individuals of the same species. So, almost all replication links are the same as in the case of cell division in both mother and father and issue. That identical base guarantees for the forthcoming forced mutation to be stable and reliable from both paternal DNA viewpoint and maternal one.

Meaning any given species, one can distinguish identical replication links such as between guanine $(\mathrm{G})$ and cytosine $(\mathrm{C})$ or between adenine $(\mathrm{A})$ and thymine $(\mathrm{T})$, what are almost all for the fertilization to be possible, from non-identical, problematic or mutational replication links featured by mismatches (such as G \& A, G \& T, G \& G, and all analogues for the rest three main nucleobases) and needing a relevant resolution accomplishable as in virtue of very complicated rules as partly randomly (even in virtue of quantum uncertainness, i.e. fundamentally randomly). The identity of all the rest DNA as belonging to the same species forces the availability of solutions in each case of problematic replication links.

Speaking theoretically, one can describe the "field of all options" possible for a certain species to change only in virtue of fertilization rather than by mutation ${ }^{12}$. The field of all options by fertilization can be described in terms of quantum and classical information in the following way to be found and founded the rules as deterministic as fundamentally random, i.e. probabilistic in all problematic replication links:

A wave function (e.g. recorded in the qubit Hilbert space) corresponds unambiguously to the species at issue therefore obeying the "conservation of quantum information" as to all individuals of the species (in the whole historical period in which the species has or had existed). Then, all problematic links can be described by the rest four binary letters (i.e. two bits) of classical information to be transmitted additionally by a backlight channel, and necessary for the speciated quantum information to be restored in any individual of the species.

Both dual qubit Hilbert spaces coincide normally, i.e. if any non-problematic case of replication links, and they does not otherwise, but only in three possible ways, which can be described by two qubit Hilbert spaces, "maternal" and "paternal", therefore necessarily entangled to each other. However then, the entanglement can be only discrete and describable by three

\footnotetext{
${ }^{12}$ Of course, the boundaries of that "field of all options" are fussy and smoothly passing into the area of options accessible only by mutations, speaking practically.
} 
options (the forth one is kept for the non-entangled state of two corresponding qubits of the "paternal" and "maternal" spaces).

In virtue of the circumstance that both wave function and single qubit represent an "infinite amount" of classical information they can be identified, and the sketched model, simplified by granting that any qubit of both maternal and paternal (thus finitely-dimensional) spaces is the same containing the identical speciated wave function. After it, any possible state of the field of all possible options for changing by fertilization featuring the species in question can be mapped unambiguously into a state of entangled maternal and paternal spaces identical as quantum information, but clearly distinguishable in relation to classical information ${ }^{13}$.

Then, still one interpretation and visualization of any species (respectively, of the process of fertilization) as a quantum calculation following the model of quantum computer suggested in a previous paper (Penchev 2020 July 9): by a program of the classical Turing machine processing a modified tape, in each cell of which the bit is replaced by a qubit.

All the three possible mutational problems after fertilization can be mapped unambiguously by three different commands of Turing machine, e.g. such as: (1) a cell forward; (2) a sell backward; and (3) go the dual qubit describing the species by the alternative nucleobases ${ }^{14}$ : $(G \leftrightarrow C) \vee(A \leftrightarrow T)$. Thus, any resolution of the problem in the framework of the model by a Turing machine program can be only deterministic excluding any quantum uncertainty unlike the real process of fertilization and suggesting one or more intermediate auxiliary mutations (i.e. processing the tape qubits) however vanishing or removed in the ultimate DNA (RNA) of the individual conceived just now.

Anyway, any species would represent rather a quasi-quantum calculation since the same qubit featuring all the individuals of the species would be recorded in any tape cell and the tape cell would remain the same during the calculation in the framework of the same species. It would transform in the general kind of quantum calculation only in the case of mutations therefore changing the speciated identical qubit in the cell corresponding to the mutated individuals.

However, the introduction of quantum calculation to describe mathematically the evolution of a given species allows for unifying "glocally" the local viewpoint of constituting an individual in the framework of a species by fertilization and the global viewpoint of constituting new species by mutations (respectively, the investigation of the transition as between individuals of the same species as between different species).

\footnotetext{
${ }^{13}$ That model implies that only two sexes, male and female correspondingly to the paternal and maternal spaces are possible in virtue of CPT-invariance being a fundamental law once quantum mechanics and special relativity are valid.

${ }^{14}$ The certain set of the several admissible commands varies in different definitions of Turing machine partly or absolutely equivalent to each other. That question is rather technical and irrelevant to the philosophical intention of the paper. The cited three commands are chosen for the substantive meaning of the interpretation of the admissible "mutations" after fertilization in terms of the model of Turing machine: the motion forward or backward means the transition to the one or the other of the pair of alternative nucleobases, and the change to the dual tape means the problem of replication link between two copies of the same nucleobase. So, the tape of that (quasi) quantum computer is the entire speciated DNA (RNA), which needs processing by a classical Turing machine program only in the case of exceptionally rare replication problems of the enumerated three kinds: after processing the standard form of a very, very long (enough for the representation as infinite to be relevant) doubled tape of a classical Turing machine is restored.
} 
The relevant proper biological terms for that "glocal" unification are "phylogenesis" and "ontogenesis" and their similarity (e.g. as von Baer's laws of embryology). However, I have used rather the mathematical model of quantum mechanics (e.g. embedded in the conceptions of local and global space often utilized for the interpretation of the "Standard model") and the two dual qubit Hilbert spaces, each of which (i.e. idempotently) is able to represent either the local or the global aspect after that unification.

That unexpected resemblance (and even maybe seeming ridiculous and doubtful at first glance) of theories in physics and biology explicable by the sharing the same mathematical model is due to their close and similar relativity to the concept of the totality able to unify definitively. Particularly, the establishment of the "natural cybernetics of time" shares the same origin from the totality explicitly (unlike the more limited and implicit approach of the original, Wiener cybernetics): time by itself orders all and thus controls anything in virtue of the prerogative delivered and provided by the totality and originating from it by the mediation of mathematics.

The sketched scheme about the application of abstract evolutionary trees can be applied to the temporal development of any subject of scientific area needing only an interpretation in the corresponding terms. Linguistics can suggest an exemplification as well:

The "first" abstract evolutionary tree of linguistics is suggested by the tree of languages united in groups, subgroups, families, etc. in a way rather similar to that of the biological evolution tree. The branches of origin are trackable analogically. A few sub-areas such as phonetics, grammar, syntax might suggest the necessary "second tree" remaining in the framework of evolutionary linguistics.

However, semantics suggests the most instructive viewpoint to the necessary second tree and even more: linkable to philosophy at least as to the Western tradition of Modernity. A potential evolutionary tree of "cognitive niches" relating to reality understood as external to language is the contribution of semantics. Both human activity and cognition advance conquering new domains needing a relevant development of language (often called the "language" of the corresponding practice or science). A force or more exactly, interaction between both trees moves language to change, and represents an image of the current extension till to the frontier of knowledge, the progress of which depends crucially on the constructed representation. Both existing language and acquired knowledge (the unity of which can be designated as cognition) constitutes a particular form of the totality doublable as reality and (or versus) language as available knowledge and (or versus) future cognition.

Thus, the lesson of semantics can be generalized in relation to the modern Western epistemology and transmitted by its mediation to the so-called mind - body problem: this will be considered in detail in the next two sections.

Though the model of abstract evolutionary tree originates from mathematics, it can be applied to itself not only considering mathematics as a historically developing area of human activity and science, but furthermore "transcendentally", i.e. meaning its special and mediating relation to the totality. Speaking figuratively, mathematics would be to be situated on the boundary itself of the "total totality" (or the totality itself therefore only a single one) and all the "particular totalities" delivering the relevant tools for them to be studied uniformly, i.e. as "hypostases" of the totality.

So, mathematics can be seen as an abstract evolutionary tree rooted in the totality and bifurcated by more and more branches in more and more detailed mathematical structures originating from each other just as the ramification of an abstract evolutionary tree. Then, two 
trees appear by themselves: the one of human practice, activity and cognition, and still one of mathematical models originating from the totality in the final analysis.

One can argue which of them is "primary" and which is derivative, in other words, which of them is actual and which is potential. As to the totality itself and being a single one in definition, that problem is meaningless, and the term of "force" (implying an ordering such as the opposition of "primary" versus "secondary") has to be complemented and equated to "interaction" (therefore excluding the former opposition): namely, a bit of information equivalent to the totality in virtue of the "fundamental, formal and logical tautology" (Penchev 2020 July 20).

In virtue of mediating across the "borderline of the totality", mathematics creates, i.e. it is able and necessary to create a structure thoroughly within itself and representing the unity of those two trees, which the separable complex Hilbert space (or respectively, the qubit Hilbert space or Hilbert arithmetic) represents. However, still one interpretation of that total (this means consistently complete) mathematical structure is possible only abandoning the claim to be total (consistently complete) or in in other words, in the framework of "Gödel mathematics"

So, but speaking rather loosely, one needs "Solomon's decision" to represent the effectively total mathematics in the modern episteme separating the totality into mathematics from the world disjunctively and gapped to each other, in a reconciling though inconsistent way. This would be a mapping of "Hilbert mathematics" into "Gödel mathematics" therefore needing "the world" in the framework of "Hilbert mathematics" being total to be represented anyway, however without including not to contradict the episteme.

That kind of solution was found implicitly by Newton and Leibniz and their infinitesimal calculation still a few centuries ago. Its essence is: reality to be included in its model effectively, by means of the change of the former and investigated by the method of derivatives (in particular, but very important: by means of time derivatives therefore reversible unlike the irreversible real time). If one paraphrases Newton and Leibniz's solution in the present context of both Gödel and Hilbert mathematics, the approach would be as if set theory be meant to be "the world" as far as set theory is the foundation of infinitesimal calculation. Arithmetic would be the corresponding image of mathematics within itself (or more precisely, in its foundations). The Gödel incompleteness of arithmetic to set theory (i.e. either incompleteness or inconsistency) is not more than an image of mathematics gapped from the world and reproduced in itself and its foundations: an image borrowed from the modern episteme as a necessary condition for mathematics to obey it in order to be admitted into it.

Now, one can reproduce the above consideration in particular, as to the "two abstract evolutionary trees" of the natural cybernetics of time. Then, the "second tree" should be interpreted as the time derivative of the "first one", and both needing a dense and smooth medium of all possible trees, what the qubit Hilbert space (respectively, the separable complex Hilbert space) represents properly. In turn, the qubit Hilbert space implies Hilbert arithmetic as

${ }^{15}$ The concept of "Gödel mathematics" (versus "Hilbert mathematics") is introduced in a rather earlier paper (Penchev 2010), but developed in a way absolutely relevant to the present context in: Penchev 2020 July 20. This is relied on two observations: (1) Gödel mathematics can be defined as that one (in fact, the contemporary mathematics) in which the Gödel (1931) incompleteness theorems are valid in virtue of involving both Peano arithmetic and set theory in the foundations of mathematics; and (2) their shared either inconsistency or incompleteness can be overcome by "Hilbert mathematics" only including the world within mathematics complementing it with a mathematical image of the world thoroughly within it. Hilbert arithmetic (being equivalent and complementary to the qubit Hilbert space) demonstrates explicitly how one can do it. 
equivalent as complementary to it; and the complementary twin of Peano arithmetic follows from Hilbert arithmetic. Consequently, the "second tree" is representable thoroughly in terms of the "second twin" of Peano arithmetic. That consideration demonstrates the consistency of the way in which the second abstract evolutionary tree is inferred as to mathematics properly.

The Lagrangian and Hamiltonian formulations of mechanics as classical as quantum ${ }^{16}$ can trace a method for abstract evolutionary tree to be introduced as to physics in a way analogical to that sketched above as to mathematics. One can describe the relation of the two "abstract evolutionary trees" in both ways: in Lagrange's and in Hamilton's manner. Both share the "first tree" identically, but interpret the second one oppositely, namely: (1) Langrange's approach considers it to be the smooth change of the former therefore involving the formalism of infinitesimal calculus; and (2) Hamilton's method achieves it only by a discrete leap. As this is well-known, only quantum mechanics unified Heisenberg's matrix mechanics and Schrödinger's wave mechanics by the separable complex Hilbert space is able to resolve the contradiction "discreteness versus continuity (smoothness)" therefore restoring the equivalence of the two formulations to each other though in a way generalizing the same equivalence in classical mechanics.

Once one has adopted the quantum viewpoint embedded in the qubit Hilbert space (equivalent to both the separable complex Hilbert space and Hilbert arithmetic), the contradiction or inconsistency of the discrete evolution (e.g. biological evolution after genetics or the empirical paleontology of fossil species) to the continuous evolution (e.g. Darwin's evolutionary theory) can be overcome, however at a certain cost involving teleological (or "reverse") causality (therefore particularly exculpating Lamarckism ${ }^{17}$ ).

One can investigate the implicit way, in which the second evolutionary tree determines anyway the conclusions of classical Darwinism. Conventionally and following the tradition ${ }^{18}$, the

${ }^{16}$ Physical and mathematical transcendentalism introduced in previous papers is applied already in them (Penchev 2020 August 17; Penchev 2020 August 30; Penchev 2020 August 31) as to the relation of Lagrangian and Hamiltonian mechanics as classical as quantum. Both formulations are as possible as equivalent in both cases and this can be inferred from physical and mathematical transcendentalism, or in virtue of the "fundamental, formal and logical tautology" following from the "postulate of the totality".

${ }^{17}$ Lamarckism can be justified by teleological (reverse) causality, for example as follows. If teleological causality complements causality (i.e. the usual one in empirical and experimental science), the discrete leap to the next generation of offspring (as in Darwinism) has to be equivalent to the continuous change of the parent generation organisms during their lifetime (as in Lamarckism). This follows from the application of quantum formalism to evolutionary theory; and not less, vice versa: the eventual empirical rejection of Lamarckism implies inapplicability. Anyway, endmost relevant Neo-Lamarckism should be probably quite different from the classical one in order to avoid empirical refusal particularly needing a real biological mechanism for the embodying of teleological causality from the lifetime changes of organism to its chromosomes in reproductive cells such as spermatozoids or ova; or suggesting alternatively the effective transmission of features to offspring by the "second" (external) evolutionary tree of environment. Indeed, the successive generations of the same species share the same environment able to deliver the same kind of training as if transmitted between generations (just according to the classical Lamarckism).

${ }^{18}$ That is: the relation of the two evolutionary trees as well as the unity of variability and heredity are meant. So, which tree to be assigned to variability and which, to heredity is conventionally and the alternative viewpoint exchanging both is equivalent. Western science and philosophy usually grant stability as primary, and change as secondary and derivative. That convention postulated to be true and obvious by the Western common sense can be tracked even to the modern Western episteme articulated and heralded by Descartes's dualism. 
second tree can be interpreted as available inwardly by variability once one has assigned the first tree to heredity. Indeed, variability can be defined also as the influence (respectively, "force" or "interaction" in physical terms) of the second tree to the first, as follows:

Variability can be juxtaposed to the Lagrangian formulation of mechanics meaning the second tree as the change or variation of the first tree. On the contrary, Hamiltonian formulation being equivalent emancipates the second tree not to be derivative and dependable on the first one. Then, Hamiltonian paradigm returned to the conception of variability suggests for it to be equivalently represented by the second tree perfectly emancipated already. Its interpretation as the evolutionary tree of environment, in turn absolutely emancipated from the standard evolutionary tree of species, implies environment to be considerable not less as the stable and primary factor of evolution (particularly, transmitting a virtual and effective "heredity of environment" to the offspring just Lamarckism ${ }^{19}$ states) just as that of the organisms themselves dwelling in it.

One can emphasize and realize the notable parallel between the sketched views in both physics and biology, two sciences absolutely gapped from each other in contemporary episteme particularly conditioning what science be in general and what physics and biology be as a corollary (and thus gapped to each other).

On the contrary, the worldview advocated here does share the same episteme though it can be tracked to transcendentalism appearing in its ground as a solution of the fundamental philosophical problem immanently irresolvable within its framework, and therefore going out of it, for example as here.

The central axiom, by which the scientific transcendentalism of the present paper differs from the dominating episteme, consists in postulating the totality not only as an unfalsifiable philosophical category grounding the being, but as a conception in the foundation of science, and thus representable in each certain science (such as biology or physics in particular) being as verifiable as falsifiable, furthermore able to unify them by shared mathematical models identifiable with reality by itself in a Pythagorean manner. In other words, the noticed analogy of physics and biology is not accepted to be accidental, wondrous, curious, and even ridiculous, but fundamental and essential, originating from the axiom of the totality and resulting into the same mathematical and mathematically modelled reality due to that reason.

Natural cybernetics of time is called to describe that kind of scientific unity, the methods to be achieved, and the foundations to be justified necessarily. Another paper (Penchev 2020 October 18) extends the same approach to history therefore outlining pathways for it to be transformed into "mathematical history". On the one hand, this means history to be mathematicized fundamentally and essentially, e.g. as physics is. On the other hand, philosophy of history is linked very closely to ontology of the totality in the Western philosophical tradition, especially and expressively, after Hegel. If the totality is granted (as in the present paper as in others similar of mine) as the ultimate and deepest source and cause for mathematization, the direct link at issue complements still a few Neo-Pythagorean reasons for the establishment of mathematical history:

They interpret as the Hegelian dialectics as the Husserlian phenomenology (including the Heideggerian and Gadamerian hermeneutics also originating from Husserl's doctrine) in the

19 The advantage of Darwinism over Lamarckism, usually granted to be confirmed empirically and experimentally very well and exaggerated as scientific truth, seems to be rather prejudice rooted in the Cartesian episteme and especially in its anthropocentrism implying "species" to be proclaimed as primary and dominating over the derivative, second obeying environment: that is an ideology of human expansionism to the world featuring the modern Western worldview at all. 
framework of scientific transcendentalism, i.e. in a falsifiable way just as the exact and mathematical sciences need it.

However, history is classified as a humanitarian and interpretative science, and it can be objective only as "historiography" registering or investigating historical facts granted to be "objective" and unambiguous, or in other words, to exist by themselves and independently of any interpretation of them. Rather paradoxically, that objectivity of historiography is a "blind alley" as to it to be transformed into a mathematical kind. Historiography has to step back to give way to the ostensibly subjective, interpretative history in order to be mathematized by a method resembling that of establishing quantum mechanics (involving the experimenter or the "apparatus" in the new kind of objectivity and being forced to generalize the objectivity of classical science, particularly borrowed by historiography).

"Historical fact" need be defined as containing some interpretation necessarily: a fact of history cannot be only what has happened (as historiography postulates), but only an inseparable pair consisting of the historiographical fact and its interpretation (whatever it be), or their unity postulated as "hermeneutical circle"20. A main innovation of Gadamer to hermeneutics (especially as a methodology of history) is the option for the equivalent substitution of any interpretation of historians post factum by the interpretation of actors, ex-ante and according to the worldviews therefore complementing the fact at issue by actors' subjective images partly predetermining their decisions and thus, the course of history resulting into a fact.

In other words, hermeneutics by itself and definitively, in virtue of the "hermeneutical circle" still one, i.e. the "second tree", the tree of "subjective interpretation" to the "objective" 21 first one withal being an equivalent temporally reverse to the second tree if it complements the first one to a "bulb-like" form what a wide enough class of mathematical lattices possesses ${ }^{22}$.

One need emphasize still one difference in order to elucidate for it to be ostensible, seeming: the evolutionary tree is actual as all species have happened unlike the virtual tree of counterfactual history, no one of which has happened. Indeed, the branches of evolutionary tree can be considered as alternative history dividing at any species as well as vice versa: any bifurcation (respectively, "historical event"), after which alternative histories appear, can be

\footnotetext{
${ }^{20}$ Hermeneutics as Heidegger's as Gadamer's does this. However one need distinguish it from the formal and logical "vicious circle" (being only a redundant tautology, and thus, useless and unable to contribute anything to a syllogism) if it is introduced in a logically consistent theory (i.e. usually "shaved by Occam's razor") what mathematical history claims to be. The "fundamental, formal and logical tautology" (Penchev 2020 October 18) demonstrates that the definition of the totality as a bit of information is a tautology of propositional logic.

${ }^{21}$ Which tree to be subjective and which one to be objective is not less conventional than the case of Darwinism versus Lamarckism as to biological evolution and discussed above. On the same reason, the tradition and modern Western episteme predetermines the one conventional alternative, namely the humans, actors of history to be granted as "subjective", "conscious", and possessing "free will" versus nature being "subjective", and not possessing either "consciousness" or "free will". However, the so-called free will theorems in quantum mechanics (Conway, Kochen 2096, 2009) demonstrate that the formalism of quantum mechanics and that of special relativity (both borrowed by mathematical history as well) implies for the two alternatives to be equivalent to each other, and thus, the choice of any of them to be conventional.

${ }^{22}$ The next several sections will investigate the way in which the bulb-like natural cybernetics of time implies the mathematical structure of lattice and then logic as immanent to thought particularly the human one.
} 
considered as a "species of historical discourse" continuing to exist by new and new descriptions in new and new historical narratives of new and new generations of historians.

Furthermore, one can admit counterfactual biological species, which might appear, but has not appeared really for different reasons, such as the absence of any accessible ecological niche relevant to their new capabilities or even only for "lack of lack" 23. The concept of abstract evolutionary tree is meant to be actual in definition therefore consisting only of all the "happy (or lucky) cases" and the "unlucky" counterfactual twins consisting of all the rest and thus, "unlucky cases" withal being "almost all" necessarily ${ }^{24}$.

One can coin the metaphor of the "lightning of evolutionary tree" in the following sense. A potential medium of all possible evolutionary trees in relation to a certain process and representable by the separable complex Hilbert space (respectively, the qubit Hilbert space) is "cut by the lightning of a real evolutionary tree" (i.e. actually taken place and observable as empirically as experimentally) ${ }^{25}$.

${ }^{23}$ The "lack of luck" can be introduced also in an absolutely rigorous way as a relation of any (virtual) subjective probability distribution to the unambiguously corresponding, objective probability distribution, or respectively as the relation of a single qubit to any more and more approximating set of bits of information, consisting of "lucky bits" (e.g. those turned out to be in the "1" state) unlike the "unlucky bits" (i.e. those in the "0" state, accordingly). Quantum mechanics is forced to assign an a priori counterpart of subjective probability to the objective probability of any natural event to happen. For example, if "Schrödinger's cat" is the case, its "dead state" can be defined as "unlucky" in an absolutely rigorous and mathematical way just as the inverse "lucky state" of "being alive". Once the separable complex Hilbert space of quantum mechanics has been involved to describe the evolutionary tree, therefore the "lack of luck" has been introduced implicitly also as well.

${ }^{24}$ The concept of "almost all" being a relation of an infinite set to a set of rigorous less power (usually the immediately next less power and particularly finite) in terms of probability theory allows for the concept of set power and even that of set to be introduced thoroughly in its scope therefore interpreting it to be the one of the two most fundamental mathematical theory traditionally representable by the pair of arithmetic and set theory and excluding it. In other words, both pairs, namely probability theory and arithmetic as well as probability theory and set theory can serve as foundations of mathematics. At last, one can imagine and describe a kind of generalized mathematics, in which all the three elements of its foundations are independent of each other: real examples belonging to it can be intuitionist mathematics, after the "middle third" being non-excluded is interpreted as a probability; or the class of non-classical logics supplied by non-Boolean lattices, in which the complement of Boolean sub-lattice to the complete lattice is interpreted as exhaustively describable by probability theory. Thus, a generalizing analogue of the Löwenheim Skolem theorem would be valid: any theory belonging to the "three-foundational mathematics" has a model in the "two-foundational mathematics" (particularly, in the mathematics grounded on Hilbert arithmetic); and vice versa. The "postulate of the totality" seems to be an equivalent of the same conjecture.

${ }^{25}$ Once the formalism of the separable complex Hilbert space is introduced to be relevant for abstract evolutionary tree to be described, the observed evolutionary tree happened really is chosen fundamentally randomly among a class of possible evolutionary trees (each of which with a different probability in general) so that the corresponding probability density distribution can be equivalently described by a wave function representing the characteristic function of that distribution and featuring the process at issue thus interpreted as isomorphic to quantum state. Furthermore, one can introduce a preceding choice for just that process to be chosen as (fundamentally) randomly as deterministically to be both observed and investigated. The discussed argument means the unity of those both stages: (1) from the separable complex Hilbert space to a certain wave function; and (2) from a wave function to an observed evolutionary tree, as a single whole; that is in the final analysis: (3) from the separable complex Hilbert space to a certain evolutionary tree. 
How can one describe all the class of possible processes transforming the potential medium (thus not only infinite but continual and smooth) into a certain actual evolutionary tree (whatever it be)?

The potential medium would be to be described mathematically by terms of both set theory and probability theory, and the evolutionary tree in question, by means of arithmetic. Consequently, the researched description is to be related to the foundations of mathematics ${ }^{26}$. Hilbert arithmetic is sufficient for it being the class of all transformations of transfinite natural numbers ${ }^{27}$ (equivalent to wave functions) into finite natural numbers, and consequently including mappings between the complementary twins of Peano arithmetic (for example the trivial identity of them belongs to the same class).

Not less, a fundamental, properly philosophical consideration of the general way for any evolutionary tree to arise is welcome in terms of transcendentalism ${ }^{28}$. Abstract evolutionary tree is the general description of the ways of how any transcendent entity (i.e. Kant's "Ding an sich") can be represented adequately in the finite scope of our human experience. Referring still once to the metaphor of lightning, the evolutionary tree cuts the "transcendent heavens" thus making them empirically visible for humans.

Summarizing, if the temporal "screen" is granted to be necessary for the description of any natural processes ${ }^{29}$, time implies a kind of natural cybernetics controlling and ordering them and describable always as an evolutionary tree therefore resolving the problem of how any whole to be divided into two halves in a fundamentally random way, which can be expressed exhaustively by the formalism of the separable complex Hilbert space borrowed from quantum mechanics (which first has been forced to find a solution of a problem belonging to the same class).

IV AN APPENDIX ABOUT THE MIND - BODY PROBLEM IN TERMS OF ABSTRACT EVOLUTIONARY TREE (BY THE MEDIATION OF THE QUBIT HILBERT SPACE)

Neo-Pythagoreanism suggests that the classical mind - body problem referring to the relation of the two fundamental poles of Western philosophy whatever they be called (e.g. "mind" versus "body", "subject" versus "object", the "ideal" versus the "material", "ideas" versus "things",

\footnotetext{
${ }^{26}$ One should mean the discussion in the previous footnote " 24 " about the linked triple of theories able to serve as the foundations of mathematics: probability theory, set theory, and arithmetic.

27 "Transfinite natural numbers" are defined as transfinite ordinal numbers (rigorously) less than any countable ordinal number and unambiguously corresponding to wave functions (Penchev 2020 July 20).

${ }^{28}$ It will be utilized in the following two sections as a link to another specific discussion about the mind body problem and Gestalt psychology

${ }^{29}$ Anyway, the theory of quantum information discusses natural processes out of the "screen" (i.e. out of time and thus out of space) such as all phenomena of entanglement, but nonetheless resulting into "dark" projections into space-time screen as both dark matter and dark energy (Penchev 2020 August 31).
} 
"words" versus "things", "signifier" and "signified", "finiteness" versus "infinity", etc. . $^{30}$ should admit a relevant purely mathematical solution, moreover being the most fundamental one.

Furthermore, the mind - body problem is not less a fundamental problem of philosophical anthropology as far as "human being" (still since Socrates as this is commonly accepted) is a main or even the most fundamental philosophical subject and cognitive puzzle, and any human being unifies "mind" and "body" (including definitively) therefore making them to be as opposed as the same (obviously a contradiction or inconsistency at least as to formal logic).

As far as Pythagoreanism was not available (and it is not even now) among the main doctrines or directions of Western philosophy in Modernity, none of the interpretations or alleged solutions of the mind-body problem does mean any mathematical formulation. On the contrary, the problem will be formulated and then resolved just as a mathematical one referring to the foundations of both mathematics and being, following the present research.

Furthermore, the same fundamental philosophical problem is quite practical for psychology as well as theoretical and refers to its foundations (being "halved" metaphorically as the city Berlin in the recent past). In the episteme of Modernity, it turns to be an "impossible science" as far as the one half belongs to the one "block" and the other one, to the other "block" being in "Cold War" with the former. That episteme of confrontation prevents any psychological research forcing it externally, artificially, and ad hoc to decide at which side of the "Berlin Wall" it is situated therefore interrupting and cutting all life ties with "relatives" on the other side. So, the unification or reunification of psychology (like that of Berlin) is necessary for it even only to be constituted as a whole as all other sciences are.

One can notice two main obstacles in psychology to be a natural science ${ }^{31}$ :

(1) It (unlike any other natural science) is forced to consider its subject as a "black box", since the investigation of how bodily and physical nerve impulses are transformed into spiritual ideas (or vice versa) is forbidden for it as far as this suggests one to "jump over the wall" (where he or she can be "shot" by the "epistemic police guarding the wall").

(2) Once the "one side of the wall" has been chosen by a researcher (e.g. that of nature here) the other side turns out to be inaccessible for any objective science. For example, the spiritual ideas turn out to be accessible only by the method of introspection and thus neither repeatable in other subjectivity nor verifiable by the researcher at issue or any other one. In other words,

\footnotetext{
${ }^{30}$ The abundance of terms about those two poles of Western philosophy demonstrates that the problem of their relation (called the "problem about the half of any whole" here) is the "basic philosophical question" (as the official Soviet philosophy and the doctrine of "Marxism - Leninism" proclaimed it) including the derivative one" "Which is primary?" The concept of information understood as a philosophical category can be interpreted also as a generalizing answer postulating itself as the answer of itself as far as the unit of information, a bit of information, being an elementary choice can be meant as an "elementary question", e.g. "“0" or " 1 "?'; 'The one equally probable alternative or the other one?', therefore being the number of bifurcations in an abstract evolutionary tree in which all branches are equally probable whether "objectively" or "subjectively" (i.e. equally "weighted" or "estimated") or equivalently: in a "Yes - No" algorithm (see Section VI). Thus, the concept of information establishes the identity of question and answer (choice) as a definitive property of the problem referring to the totality and originating from the totality itself (particularly from its definition to "be all" including both answer and question simultaneously).

${ }^{31}$ The analogical and opposed obstacles in psychology to be a humanitarian ("spiritual") science are not less, but they are not a subject of discussion in the present paper according its intention: natural cybernetics of time.
} 
natural psychology cannot satisfy the criterion of falsifiability in relation to any idea fundamentally, being situated on the side of nature to the "epistemic wall".

Maybe Husserl was that philosopher and scientist who approached more closely to a relevant solution of the problem about the foundations of psychology (starting from, but overcoming the contemporary episteme of knowledge or possible cognition). He was a mathematician by education who entered philosophy trying to justify arithmetic (in turn seeming to be the base of mathematics) psychologically (1891). However, he saw rather soon that he came upon the "epistemic wall" preventing psychology and thus his attempt to justify arithmetic by it.

To overcome that fundamental obstacle he was forced to rediscover transcendentalism by his own postulates and methods (such as 'epoché' or 'psychological reduction', its logical and mathematical correlate of 'eidetic reduction', its properly philosophical correlate of 'phenomenological reduction'; and at last, their unity as 'transcendental reduction' therefore establishing a new field of philosophical research, namely 'phenomenology' recoining a then existing term, but in a fundamentally different sense).

Of course and as it is commonly accepted, that is a breakthrough located in the foundation of the $20^{\text {th }}$ century's philosophy and predetermining it essentially. However, only a much more particular application of it will be emphasized now and referring to the unity of psychology and mathematics (inferable, for example, from the phenomenological unity of psychological reduction and eidetic reduction) as a necessary condition for psychology to be justified overcoming the existing "epistemic wall", which is often articulated as the well-known mind body problem:

'Epoché' in an only psychological sense calls for identifying the entities in either "side of the wall" once the question to which side any entity belongs has been abandoned in relation to all psychological "phenomena" (as in Husserl's sense as in the usual one): 'psychological reduction' can be understood as that only psychological epoché (i.e. referring only to the area of psychology and to the consistent way for it to be established overcoming the preventing obstacles of the contemporary episteme). One can notice that 'psychological reduction' is a method transferring the philosophical transcendentalism in a particular scientific region, namely psychology. It shares that "doubling" is specifically transcendental and features any form of transcendentalism. Thus, but implicitly, all the black box (as well its elements) meant as the fundamentally hidden subject of psychology until then is postulated to be accessible constructively.

Using the contemporary terms, those elements being constructively accessible would be to be called "bits of information" and the "black box" of psychology would be to be constructive and accessible by the theory of information. Furthermore, "psyche" investigable naturally (i.e. by psychology as a natural science) would be to be understood as information respectively as both quality and quantity.

Eidetic reduction, in turn, means the usual general approach to be obtained an "idea" (particularly, a formal and mathematical notion) from anything being "material" by removing all variable (acceptable as "accidental") properties or relations, being an infinite cardinal number in general, remaining in the final analysis a nucleus of invariant properties or relations, almost always being a natural number (i.e. finite in Peano arithmetic), called also "essence", "eidos", "definition", "idea", etc.

In the present context and following Husserl's intention (1900 \& 1901) not to justify arithmetic by psychology (as he had attempted in 1891's paper), but on the contrary, psychology by generalized mathematical methods (what eidetic reduction is in fact and properly), one need 
unify psychological and eidetic reduction therefore appealing to phenomenological reduction (and thus, to transcendentalism as far as phenomenological and transcendental reduction coincide with each other formally),

Using again the metaphor of the "wall" (still more that it contains a formal structure shared by the mind-body problem and a bit of information), the process of eidetic reduction defines a surjection of all "things" on the "one side of the wall" into all "ideas" on the "other side of the wall" (respectively, a set of injections reversely). However, the "psychological reduction" (i.e., by the same metaphor as well as in the same terms) postulates a bijection between them. This implies ostensibly (fortunately, only at first glance) for psychological and eidetic reduction to be unified consistently. In fact, it is a paradox only to common sense, but not to formal logic. The alleged seeming obstacle consistc in the bijection (for psychological reduction) of a set of finite sets and a set of infinite sets (i.e. an implicit surjection for eidetic reduction). This is not a contradiction for bringing arguments into three hierarchical levels:

1. Bijection is a particular case of surjection (where the set of reverse injections consists of a single element).

2. The set of finite sets (i.e. on the "ideal side") may be infinite (just as the set of infinite sets, i.e. on the "material side" may be finite. e.g. as the set of all entities in the universe being postulated to be finite).

3. The concept of set-theoretical finiteness (more known as "Dedekind's finiteness") allows for a generalized bijection ${ }^{32}$ of the elements of finite and infinite sets.

Either of both pairs $(1 \& 2$ or $1 \& 3$ ) defines a methodical pathway for the alleged contradiction not to be formal and logical. The former $(1 \& 2)$ is followed by Platonism and all contemporary (including transcendentalist) versions of it simply doubling all things by corresponding ideas unambiguously. However, Husserl emphasized permanently that his phenomenology is out of the scope of Platonism. So, the later ( $1 \& 3$ ) should be suggested rather in relation to the method of the phenomenological reduction as uniforming both psychological and eidetic reductions.

That alternative of Platonism may be ("quantum") Neo-Pythagoreanism thus sharing the formalism of the qubit Hilbert space (respectively, "Hilbert arithmetic") and as the present paper advocates. That option can be justified as follows:

An intermediate link is the concept of quantum information once its equivalence to the classical information of infinite series or sets has been proved (Penchev 2020 June 18). Then, the result after eidetic reduction can be a single qubit in the final analysis, furthermore, doubled by a dual qubit for psychological reduction. If one means all possible "things" and their "ideas" corresponding unambiguously and being an infinite set in general, the qubit Hilbert space (respectively, Hilbert arithmetic or the separable complex Hilbert space) would be that model relevant for mathematical psychology after Husserl's revolution, namely: psychology to be justified by arithmetic (logic) rather than vice versa (according to his initial intention in 1891).

\footnotetext{
${ }^{32}$ That generalized bijection grants that the infinite set at issue is mapped into an arbitrary finite set being chosen fundamentally randomly among the set of all finite sets in any trial of bijection of that kind therefore defining unambiguously a probability distribution of the set of all finite sets rather than a certain finite set as the bijective image of the infinite set at issue (Penchev 2020 July 20).
} 
Granting that, any psychological state of the world (i.e. the world represented in one's mind) is a certain wave function, i.e. isomorphic to the same world by itself ${ }^{33}$ : an observation made still by Schrödinger (e.g. in 1935's paper).

The second objection ("(2) Once the "one side of the wall" has been chosen by a researcher (e.g. that of nature here) the other side turns out to be inaccessible for any objective science" above) can be overcome by mathematical psychology in a Husserlian manner. Furthermore, one should demonstrate what the "black box" meant in the objection "1" contains, or what the elements of mathematical psychology look like, being information and measurable in bits accordingly: well, neural networks and thus isomorphic to abstract evolutionary trees.

Indeed, one can see the separable complex Hilbert space (or its qubit equivalent) as an infinite, dense, and smooth medium containing all possible neural networks including infinite ones, which can be researched only mathematically rather than observed empirically or experimentally. Thus, that Hilbert space contains as a (finite) subset all real neural networks. Here is how any real networks being an element of that subset is able to link both "sides through the wall", i.e. to be what a "black box" contains:

The two dual Hilbert spaces can be interpreted correspondingly as the "wave function of consciousness" and the "wave functions of reality" coinciding to each other in the hypothetical (or properly mathematical) case of infinite neural network; and vice versa: any real, both finite and loose, reticulate (i.e. not dense) neural network implies a certain nonzero difference between the "wave function of consciousness" ("mind") and the "wave function of reality" ("body"), or a relevant entanglement distinguishing each other ${ }^{34}$.

In other words, the solution of the mind-body problem in the present context can be the following:

Human brain is a medium, in which a huge number of real neural networks can take place. Each of them means a state of reality and a state of consciousness linking them by itself and representing their "difference". Thus, the bodily side is the one dual "half", and the mental side is the other dual "half" of the same whole meant implicitly by the neural network at issue.

The denser and more extended is a neural network, the less is the difference between its two sides (e.g. "body" versus "mind"): i.e. the more adequate is that neural network. The cognitive progress (e.g. that of an individual or that of a science) can be expressed by diminishing that difference due to the conservative ${ }^{35}$ development of the neural network identifiable as the same (i.e. in relation to the same part of reality).

33 The same isomorphism is often interpreted incorrectly, as an alleged dependence of reality on consciousness once the contemporary episteme for them to be gapped has been granted. In fact, their opposition is relative and invalid as to quantum mechanics (or as mathematical psychology as one can sees, or at least as the present paper advocates) where they are unified by an underlying mathematical isomorphism interpretable equally well as the wave function of the state of consciousness as the same wave function of the state of the world.

${ }^{34}$ On the other hand, a certain real neural network can be related to a certain curving of Euclidean space expressible by the mismatch of the relevant contravariant and covariant space and thus, by a state of pseudo-Riemannian space. A mapping of entanglement and pseudo-Riemannian space exists under certain conditions and it can be interpreted as an entanglement theory of quantum gravitation (Penchev 2020 August 31).

35 However, jump-like reformations of neural network (thus non-conservative) can happen being conditioned by the law of maximal entropy particularly as the slowest increase of information (the "principle of least choice" in: Penchev 2020 October 18) after the development of neural network as the jump-like reformation (if possible) to a state of less information. 
Thus, neural networks can be adopted as the relevant element of mathematical psychology. They are contained in any "black box" linking the bodily and mental sides in the mind-body problem in the framework of the modern episteme established still since Descartes's dualism.

Each real neural network can be represented topologically equivalently by an algorithm containing "yes - no" bifurcations and then, by the well-ordered tape of Turing machine able to fulfil it (as this will be discussed in detail in Section VII). If one postulates that the algorithm and correspond tape of Turing machine are equivalent and contain the same quantity of information, this implies particularly that the choice meant as deterministic in any "yes - no" bifurcation and the (well-) ${ }^{36}$ ordering of any two cells as equivalent as containing the same amount of a bit information. That observation can be granted as a particular case of the equivalence of the axiom of choice and the well-ordering "theorem" in set theory.

Furthermore, neural network means a certain whole and thus, its two dual sides, by means of two possible ways: the infinite neural network can coincide with the whole; the real neural network is only related to the whole or designates it. That whole correlates to the neural network and can be interpreted as Gestalt in the namesake psychological theory or doctrine. Whether the elements of psychic are accepted to be neural networks or Gestalts is equivalent and just this will be discussed in the next section.

\section{THE CONTINUATION OF THE APPENDIX: THE MIND - BODY PROBLEM AND GESTALT PSYCHOLOGY}

Though Gestalt psychology is a large field including many and most various results, only its fundamental postulate (Lewin 1936) will be related to mathematical psychology: psychology (one might complement: like thermodynamics or quantum mechanics) is holistic definitively: its primary elements are "Gestalts", i.e. those kinds of "whole" In other words, the constitutive viewpoint to psychology is "outside" as far as the wholeness is visible directly and explicitly just outside. On the contrary, remaining inside, one need postulate it just as philosophical transcendentalism has to postulate

the generalizing conception of the totality being visible empirically or experimentally only from the hypothetical and inaccessible viewpoint "outside", the alleged "viewpoint of God".

So, "psychic" after Gestalt psychology can be realized as a particular form or interpretation of the philosophical (or even theological ${ }^{37}$ ) totality, therefore implying "mathematical psychology" as far as mathemazablity follows the axiom of the totality.

The wholeness of Gestalt is able to overcome the (2) obstacle in the previous section, preventing psychology studying mental states (or "spiritual ideas") by introspection to be an objective science (e.g. as physics). Any mental state is already accessible objectively by the "wave function of conscious" (furthermore definitively coinciding with the "bodily wave function of reality") and embodied (i.e., again bodily) in a brain state (i.e., in a real neural

\footnotetext{
${ }^{36}$ This is bracketed because the ordering of two elements is always well-ordered, and thus, that word of "well" is redundant.

${ }^{37}$ The reference to the totality as theological, i.e. to "God", rather than as philosophical, is even more relevant as to Descartes, as far as his dualism constituted philosophy rather as opposed to the totality meant definitively by theology. On the contrary and following the conception of scientific transcendentalism, one can generalize Husserl's idea about "philosophy as a rigorous science" into a "theology as a rigorous science" defining it as a science studying the totality (necessarily postulated) and therefore "bracketing" the millennial opposition of theology and science (but not that of religion to science) in Husserl's "phenomenological manner".
} 
network) representing both "body" and "mind" by their mismatch, i.e. as a certain finite difference.

One can discuss particularly a reverse reflection from a real finite neural network (apropos, always representable by an algorithm or by the work of a Turing machine) to consciousness, or "mind" (versus "body), properly attachable only to infinite neural network, never existing really: empirically or experimentally for psychology. On the other hand, the consciousness (or "mind") is obvious for any human being "inside", though his or her mental states (respectively, neural networks) are definitively finite being exemplified by the finite brain states. Thus, an annoying contradiction appears seeming able to discredit the applicability of mathematical psychology to Gestalt psychology. It fact, it is not only surmountable easily, but hints a solution of the problem whether "computer can think":

The solution is as analogical as isomorphic mathematically to that of the so-called sorites paradox: adding more and more links in a neural network one by one (similarly to grits, meaning sand grains, added one by one to an available and spatially limited collection of them and transforming them into a heap, respectively "Gestalt" or "wholeness", by themselves or by itself gradually) acquires "consciousness", respectively "mind" more and more probably, i.e. "flattening vertically" the corresponding probability (density) distribution more and more after each added "grain" (i.e. a new neural link). One may say that the real finite neural network of a human being is extended enough, so that it implies for the existence of consciousness to be available "almost always" (mathematically) or always practically.

The solution of the problem whether a computer thinks suggests the Kolmogorov complexity of algorithms accomplishable by it to be comparable to that of human neural network in average (even only mathematically). The comparison meets a few fundamental obstacles:

One would be to mean the dynamic complexity (i.e. trainability or the capability of self-organization) of a computational medium, or the computer's ability to change its algorithm in order to achieve a certain finite state (i.e. the solution of a certain problem). Trainability multiplies complexity therefore needing a much more concise algorithm (however changeable in virtue of a meta-algorithm, the complexity of which is the "factor of multiplication") to achieve the same solution in comparison with an unchangeable algorithm.

The real human neural network represents the propagation of electric and chemical impulses gradually in time, however coexisting as a whole in the shared area of irradiation and neuro-electrochemical excitation. On the contrary, all computer algorithms including self-training are always representable by a single well-ordered series of Turing machines. Speaking loosely, neural network means Gestalt directly, but it has to be represented equivalently as a temporal computer sequence.

Namely this implies for the mathematical model of quantum mechanics to be borrowed by mathematical psychology needing just as quantum mechanics to represent Gestalt as well-ordered temporal series equivalently once the model of neural network has been involved.

One can notice the following, possibly heuristic ambiguity or fusion. The real human neural network exemplified by a brain though being finite is a whole in virtue of its simultaneous irradiation. Nonetheless, the relevant abstract neural network (which is representable properly by an algorithm whether self-training or not) implies the existence of a relevant wholeness in the probabilistic degree, in which the finite abstract neural network can be considered as an infinite one mathematically. 
Thus, the real human neural network seems to be a whole even finite, but the abstract neural network representable by a relevant finite algorithm can be a whole only probabilistically. So, the former seems to be holistic absolutely, i.e. by itself, unlike the latter being holistic only relatively, i.e. only in relation to its infinite counterpart.

In fact, the alleged opposition is ostensible and it is due to the following. The real human neural network is a whole only "almost always" mathematically being complex enough permanently. So, it is a whole by itself only empirically or experimentally (as far as any human experience or experiment is finite definitively), but not mathematically, and thus, not absolutely. On the contrary, it is relative, just as an algorithm developing successively thinking in a rigorous mathematical meaning.

The "Turing test" by the isolation in a "Chinese room" is well-known, and even glorified. One can notice that the checked "human intelligence" of the investigated computer is only hypothetical just as the validity of any scientific theory: a suggestable future experiment can distinguish the answers of the machine from those of a human being.

In fact, if one tries to imagine how the test might be accomplished practically, many ambiguities would appear ${ }^{38}$. In fact, it would not be to be interpreted as an experiment whether real or thought, but rather as an exemplification of the modern episteme as to the problem whether a machine can think in the sense implied by that cognitive disposition ${ }^{39}$.

Indeed, the "Chinese room" substitutes the "black box" used explicitly in the present paper to describe the fact that dualism of mind and body hides definitively any possible mechanism of their interaction according to the cognitive episteme of Modernity. That "Chinese room" mediates the answers of a human being as a hypothetical standard, on the one hand, for the investigated series of answers, on the other hand. Both series are to be identified or not without entering the "Chinese room", respectively, the "black box" juxtaposing any body and any mind including those eventually belonging to the same human being or to the same machine.

Thus, the "Chinese room" of the Turing machine suggests two "black boxes" to be compared and then identified or not. The present paper adds a very probable conjecture about the same "substance" contained in both "black boxes" namely neural networks (or more precisely, a finite set of finite networks variable in time in virtue of the property "trainability"). A quantity, such as Kolmogorov complexity (interpretable furthermore as the information of a set of well-ordered series) can unify them suggesting that a threshold would exist, after which consciousness should appear.

However, the utilization of the separable complex Hilbert space of quantum mechanics needs that threshold to be probabilistic (both analogue and isomorphism to ,tunnel transition" are relevant). In other words, a "potential barrier" surmountable probabilistically, i.e. fundamentally randomly, exists distinguishing any human being from any computer (being representable as a Turing machine). The more complex are the algorithms accomplishable in a computer, the more

\footnotetext{
${ }^{38}$ For example, how might one establish that a certain answer of the computer will be not answered by any human being ever?

${ }^{39}$ One can take an analogical test, after which a human being claiming to be "God" is isolated in a Chinese room and questioned about whatever. God is postulated to answer correctly any question, so the test at issue would fail after the first wrong answer. That modification of "Turing test for God" demonstrates that the test suggests rather a definition of thought than a real test of thought according to the literal meaning of the utilized term "test": it means the class of all possible tests here rather than a certain test applicable really.
} 
probable it is to overcome a Turing test and to possess consciousness (for example, as a necessary condition after a certain degree of trainability).

Thus, the intelligence of human beings is postulated as a practical standard as possessing consciousness "almost always" (i.e. admitting for any non-pathological human being in an awake state not to possess consciousness in negligible cases and less than any machine).

One can suggest an alternative, the semantic description of the way for a human being and a machine to be compared about whether they think (even regardless of whether the human being at issue is granted to think or not). That is a criterion interpretable as a solution of the mind body problem establishing a conventional semantic boundary in the mind - body continuum.

Semantics, presumably first (even before quantum mechanics and psychology) among the sciences possible in the modern cognitive science: in the end of the $19^{\text {th }}$ century, had been forced to overcome the mind-body gap, however assisted by a peculiarity specific for semantics and which will be discussed in the next paragraph:

Semantics introduced "sign" unifying "signifier" and "significant": the last two notions sharing the same "bodily" side of the dualistic abyss. Those are correspondingly the "words and things" both empirically or experimentally accessible according to the classical paradigm of objective science. Thus, the other, "mental" side is only marked implicitly in the "sign" and then right away "bracketed" in virtue of the internal and hidden identity of the sign involved just for postulating that identity largely.

The innovation of semantics can be interpreted as follows. The correspondence of the hidden "mental image" accessible only by introspection and thus not to "objective science", on the one side, and the "objective thing" accessible empirically, on the other side, to be substituted equivalently with two disjunctive kinds of objective things, the one called "words", and the others, "things" only implicitly intermediated by the inaccessible "mental image" and conjectured as the class meant as "sign". Only the foundations of semantics need the concepts of sign in order to link the "words" and the "things" tightly: it is not more than the class of relations of them.

If one has already connected the introspective mental image corresponding to the objective thing somehow, by "black box" or explicitly, by neural network as above, this has only added a second neural network in opposite direction to the disjunctive second kind of objective things therefore called otherwise (e.g. "things" versus "words"). The composition of both neural networks constitutes a complex neural network contained in the "black box of sign" however relating only to empirically accessible things though fundamentally different and distinguished: thus the pathway to the objective research of the complex neural network at issue is open.

Thus, neuro-semantics discusses any sign as a class of neural networks able to relate any investigated thing to its relevant word. The consideration until now supposes human beings, to whom terms of mental images, things, words, and neural networks (in a narrow and literal meaning) make sense.

Now, the same description will be utilized as to computers as well. The problem will be: what is the abstract necessary condition for the concept of mental image to make sense to computers not worse than to people?

The conclusion which is forthcoming to be justified is the following:

One can grant "mental images" in any computer after doubling the "words" processing in its program admissible for its software by corresponding "things" out of the computer and meant by relevant words. A syntax generalizes all the class of rules of how words to be processed, but it does not need "mental images" in general and Occam's razor would "cut" them. On the contrary, 
even a rudimental or rudimentary semantics implies them. So, the initial problem is able to be reformulated: which is the minimal threshold implying for any semantics to exist necessarily as to an arbitrary system (such as a computer) preferably represented formally (e.g. as Turing machine or quantum Turing machine)?

The occurrence of semantics definable as the doubling of purely syntactic "words" by "things" as their semantic counterparts is not more than still one form of transcendentalism, now linguistic. So, semantics and syntax are to be identical as to any infinite neural network (and mathematically embedded in the qubit Hilbert space), but different as to any finite one such as those in any human brain (or respectively, in any computer after representing any neural network as an algorithm equivalently).

The alleged "thinking computer" (being defined by an eventual nonempty semantic projection $^{40}$ ) is intermediate between actually infinite neural networks and finite ones just as any "thinking human being", i.e. thinking in definition. All intermediate positions are occupied by "quantum computer" as far as the state of which is a transfinite natural (ordinal) number. However, all real human neural networks are finite "bodily" (as composed by real neuron cells in the brain being a real human organ), but nonetheless, infinite "spiritually". One need realize the constructive way for that contradiction to be reduced to the unobjectionable duality or complementarity in order to serve as a paradigm of "thinking computer" to be modeled.

Self-consciousness being a doubling of consciousness is a testimony of its occurrence. Neural network need double itself somehow, by which it is able to refer to itself as a whole (i.e. wholeness, an exemplification of the totality ${ }^{41}$ ), and after which it can interpret itself analogically as infinite and in an absolutely consistent way to the bodily interpretation as a state of the human brain.

That doubling as to a computer or algorithm can be exemplified by a meta-level (e.g. as after resolving the "halting problem" proved irresolvable in the level of Turing machine, itself, but rather trivially resolvable by adding any "observer" or meta-level) and referring to the level at issue definitively. The occurrence of meta-level seems to be unstable and fundamentally random (probabilistic) permanently threatened to vanish though appearing soon again. One can admit that there might exist algorithms as well as computational methods and techniques assisting or preventing the occurrence, establishment, fixation and validation of a computer's consciousness following directly or not anthropogenesis as the single known successful example of neurogenesis and the generation of spiritual dimension.

\footnotetext{
40 That "thinking computer" can be defined by two independent syntaxes unified in any way, e.g. entanglement, rather than by the new dimension of semantics; in other words, the second dimension whatever it be, even still one syntactical (respectively the occurrence of that dimension is essential rather than its kind to be semantical, syntactical or whatever else0.

${ }^{41}$ Religion investigated as an anthropogenic factor available in all human societies and civilizations though contradicting empirical or experimental scientificity can be justify by the above requisite: God (respectively the abstract divinity or deity) means an external (or doubling) viewpoint to empirical experience therefore implying self-consciousness and the second "spiritual" dimension necessary for thought. Thus, religion from the viewpoint of anthropogenesis or neurogenesis seems to be a social practice assisting all individuals in establishing and fixing the validity of spiritual dimension, a fundamental feature defining human beings. It might be interpreted as a "Wittgenstein ladder" in relation to thinking and consciousness, not necessary and removable once human being is reasonable already steadily.
} 
The problem of whether a natural boundary of neural complexity, after which consciousness appears necessarily, exists is open as well.

\section{AN APPENDIX TO THE APPENDIX: ALGORITHM IN TERMS OF} EVOLUTIONARY TREE

An objection about the equivalence (respectively, mutual interpretability or applicability) of "algorithm" and "evolutionary tree" (also in terms of Feynman trajectories) can be the following. The branches of algorithm are alternative and only one of them is real as to a certain calculation, and those of an evolutionary tree are accomplished simultaneously in parallel, thus all of them are real and implying a relevant probability (distribution) for each (or all) to occur. The corresponding Feynman trajectories share the same probabilistic description interpreting the same "probability" as quantum (i.e. as both subjective and objective) rather than as only objective after a real evolutionary tree. While evolutionary trees and Feynman trajectories share their common mathematical structure, the algorithm does not at first glance or need a relevant consideration for that structure to be generalized as to it.

Indeed, if one means the class of all performances of the same algorithm, this implies a certain objective probability for each branch of it to take place. Different algorithms might share the same branch with the same probability. So if an evolutionary tree is given, a class of relevant algorithms would correspond: an algorithm (just as a real evolutionary tree) is representable as an interpretation of an abstract evolutionary tree.

In other words, two distinctions (or generalizations) can mediate the transition from "algorithm" to "evolutionary tree", and then, to "Feynman trajectories": (1) any deterministic bifurcation of an algorithm is interpretable as a corresponding probabilistic branching of evolutionary tree, after all performances of the same algorithm; and (2), any evolutionary tree corresponds to a quantum state (represented by Feynman trajectories) after substituting objective probability by quantum probability.

However, the ultimate objectivity is the unification of "well-ordering" and "coherent state" via "partial ordering". It can be distributed in a few stages: (1) the bidirectional link of well-ordering and partial ordering by the work of Turing machine; (2) the discussed here and above the bidirectional link of "partial ordering" (as "evolutionary tree") and coherent state. An additional motivation is (3) the link between "partial ordering" and "lattice" (as the algebraic structure corresponding to logic or to the class of logics).

Only (1) and (3) will be inferred now as far as (2) was elucidated already above.

(1) Here is a constructive procedure of how one can reorder a partial ordering to well-ordering or vice versa, implicitly involving the paradigm of calculation by Turing machine:

A branch of partial ordering is chosen to be first under the condition to contain a least element in the set of all least elements, one for each branch. The evolution tree can be defined under the condition to contain a single least element (thus corresponding to some lattice or lattices). Then, any partial ordering can be continued hypothetical to a relevant evolutionary tree originating from a single progenitor ${ }^{42}$. One can mean that evolutionary tree rather than the partial ordering at issue. The equivalent well ordering will be found for the evolutionary tree, after which all added

\footnotetext{
${ }^{42}$ Furthermore, one need prohibit any merging of different branches, which takes place in a lattice. However, this is easily resolvable conventionally. Any segment shared by two branches after their bifurcation can be doubled so that each copy will belong only to just one of them. Following that conventional rule, one can represent the partial ordering as an evolutionary tree.
} 
complements of the branches of partial ordering will be removed so that the last element before the cut segment and the first element after it in the ultimate single well-ordering to be successive immediately (nextly).

The availability of a relevant program in any Turing machine supplies a bijective correspondence of its tape into the tree of its algorithm. The relevant subprograms process the beginning and end of each branch in a way to order them well (i.e. not partially as in the original algorithm tree). This turns out to be possible since any tape cell (in which either nothing or " 0 ", or " 1 " can be as written as read) can be considered as an elementary bifurcation (or "branching") and then any tape cell bifurcation and any tape cell successor can be equated in virtue of the fundamental equivalence of the axiom of choice and the well-ordering "theorem" in set theory (i.e. the equivalence of "choice" in a bit and the well-ordering of two bits after which the one is the "successor" of the other). Particularly, the tape of the Turing machine consists of the successive alternation of those two forms of the same (both bifurcation and ordering).

Then, the problem is whether any well-ordering can be identified with a certain tape of the Turing machine. The aforementioned fundamental equivalence in set theory implies for them to be identifiable absolutely under that condition.

The conclusion is that a partial ordering and a well-ordering are equivalent under the following two conditions: (1) the equivalence of the well-ordering "theorem" and the axiom of choice as in set theory; (2) an additional description supplied by the relevant subprograms and complementing the algorithm at issue to just one tape well-ordering. In other words, "partial ordering" means a class of equivalence of "well-orderings" (each of which representing the same partial ordering by means of a different program, and thus, by a different Turing machine ${ }^{43}$ ).

Jumping over (2) elucidated already, one passes to (3) directly:

The partial ordering eventually complemented to an evolutionary tree (if need be) can be doubled to be transformed into a lattice, and thus, an evolutionary tree can be considered as the "half" of a relevant lattice. The sense of that doubling in relation to any evolutionary tree (as any evolution is meant as a process in time) can be understood in two "mirror" temporal ways: (1) as the same evolutionary tree going in the opposite direction of time; (2) as the same evolutionary tree represented in a "mirror of time", and thus, in the same direction of time so that that it complements the evolutionary tree at issue to the relevant lattice ${ }^{44}$.

43 This corresponds to the well-known and even trivial fact that any algorithm can be processed by different programs.

${ }^{44}$ One can investigate the influence of one or another property (such as the idempotency of negation, commutativity, associativity, distributivity, or modularity of logical operation) to the kind of corresponding lattice. The idempotency of negation implies for the lattice to be complemented: only this property is discussed above. The others impose additional restrictions to the graph connections of any pair of elements (commutativity) or to any triple of elements (associativity, distributivity, or modularity). Those restrictions might be related further to the evolutionary tree substituted by a lattice, but they do not seem to possess any intuitive sense or visualization. In other words, the algebraic notation for logical properties, relations or operations is preferable as most concise. On the other hand the eventual meaningful interpretation of them in terms of neural networks can be very instructive and useful. The logical laws or axioms (being forms of conservation) impose universal (i.e. repeating) local symmetries (lattice patterns). A relevant generalization (or application) of the conservation - symmetry theorems of Emmy Noether seems to be possible. Another interesting problem might be what the relevant lattice patterns (symmetries) of logical laws or axioms (conservations) would imply for the structure and functioning of a neural network. 
As a conclusion, any algorithm (respectively, the work of the Turing machine) can be realized as an evolution tree of processed calculation.

\section{TIME BY THE ABSTRACT EVOLUTIONARY TREE}

Time is a fundamental physical quantity and sometimes considered as the universal variable of any change not only physical (i.e. "bodily"), but not less mental (i.e. referring to "mind" in the mind - body opposition). Thus, time as variable occupies a unique place in the modern episteme (since Descartes's dualism) being able to unify those "body" and "mind" therefore being even a subject of philosophical investigations (such as Heidegger's "Sein und Zeit"). However, that unique property is at the cost of "time arrow" forcing the other "half of the world", i.e. in the opposite time arrow to be "lost" as inaccessible empirically or experimentally".

As to the class of very well-confirmed contemporary physical theories, they can be divided into two groups as disjunctive to each other as exhaustive to all physical theories, according to the mathematical interpretation of the concept of physical time. The one group considers it to be reversible just like all other physical quantities. The irreversibility of time is complemented externally to the mathematical structure of the corresponding theory and can be considered as an implicit additional consistent and independent axiom to it or not less, as necessarily added by the implementation of mathematical structure (neither empirical nor experimental) to the real physical theory being experimental and empirical and referring only to processes and phenomena in time. Classical mechanics, special and general relativity as well as many other more specific theories belong to that group.

The other group includes the irreversibility of time in the conceptual base of the theory at issue, from where it is embedded in mathematical forms serving it. All thermodynamic theories and quantum mechanics ${ }^{46}$ belong to it. The thermodynamic theories involve a new fundamental physical, but physically dimensionless quantity of entropy and specific for them: featuring only them. Quantum mechanics is reformulable equivalently and exhaustively as theory of quantum information, in which "quantum information" can be defined as a physical quantity related or generalizing physical entropy, on the one hand, and can be considered as a generalization of the classical concept of information referring to infinite series or sets (Penchev 2020 June 18), on the other hand. Besides by introducing "entropy", the physical theories of irreversible time can be featured or defined in a few ways independent of each other only at first glance:

(1) All of them are holistic necessarily, though as explicitly (statistical thermodynamic theories) as implicitly (phenomenological thermodynamic theories and quantum mechanics). The former kind elucidates the mathematical links between the statistical ensemble of elements and their probability (eventually, density) distribution, on the one hand, and the phenomenological (i.e. in the sense of "holistic" rather than in that of Husserl's phenomenology) behavior of a relevant whole resulting in specific thermodynamic quantities (entropy is one among them), on

\footnotetext{
${ }^{45}$ Anyway, the "other half", i.e. in the opposite time direction would be not to vanish, but to be available somehow among the physical being in some form though its link to the opposite time direction not to be well-known (or to be problematic): for example, as Dirac's antimatter or even as the standard matter after general relativity as the commonly accepted theory of gravitation (Penchev 2013). A formal way (but not less, fundamental and philosophical way for the being to be generated by dividing "nothing" into two half (therefore implying for being to be "less than nothing") is discussed in another paper (Penchev 2019).

${ }^{46}$ Quantum mechanics can be interpreted as a thermodynamic theory of mechanical motion, or respectively, as a thermodynamic generalization of classical mechanics, but in a way fundamentally different from that implementable in statistical thermodynamic theories.
} 
the other hand. The latter kind describes only the observable whole without referring to any hypothetical elements. ${ }^{47}$

(2) All of them suggest an external observer. The "apparatus" in quantum mechanics can be considered as an objective "external observer" for the fundamental Planck constant dividing it from the measured quantum entity with a finite (though extremely small) physical action. Boltzmann's thermodynamics (also Gibbs's or Einstein's one as far as they are equivalent to it or can be considered as partly equivalent to it) admits an implicit "internal observer" able to measure the mechanical quantities of elements as well as to establish transformations after changing the viewpoint of an internal to an external observer or vice versa.

Quantum mechanics also allows for an implicit internal observer in virtue of Niels Bohr's postulate of what it studies: namely, the quantum system of both investigated microscopic entities and macroscopic apparatus only by the readings of the latter. If the external observer be identified with the readings of the apparatus (respectively, with the observer, human researcher only registering them unambiguously), the internal observer would be to be admitted implicitly or hypothetically as perceiving directly coherent quantum states (namely those of the quantum whole of the entity + apparatus or of the entity by itself). However, the physical actions of that internal observer as to quantum mechanics would be restricted not to exceed the Planck constant definitively: otherwise being transformed into an external observer immediately and necessarily.

Consequently, that internal observer might be only hypothetical as far as the sub-Planckian area is postulated to be not accessible experimentally and still much less, empirically. Bohr's postulate implies for the transformations between the hypothetical internal observer and the real external observer to be a bijection to conserve the necessary objectivity of quantum mechanics as an experimental science, but a generalized kind of bijection, which may be called "transcendental bijection":

Though the term "transcendental bijection" is coined here for the first time, the corresponding idea has been developed in previous publications (Penchev 2020 July 20). Its essence is "transcendental doubling" or "transcendental invariance", both due to the totality and interpretable formally by any structure isomorphic to a bit of information. For example, a relevant and suitable visualization of that transcendental bijection is the bijection of the Turing machine tape cell, once, empty, and twice, being recorded (whether " 0 " or " 1 "). However, unlike the usual sense of bijection, it admits to be represented also as a bifurcation (either " 0 " or " 1 "); or in other words, a generalized kind of identity including bifurcation is introduced.

In fact, the totality (respectively, any wholeness which can be considered as a "particular totality", or the totality in a certain particular relation) forces that generalization of identity. This

\footnotetext{
${ }^{47}$ That statement needs an explanation as to quantum mechanics, which should mean the theorems of the absence of hidden variables in quantum mechanics (Neumann 1932, Kochen, Specker 1967). Those theorems imply that elements analogical to those in statistical thermodynamics cannot exist once the mathematical formalism of separable complex Hilbert space has been granted to be relevant. Those non-existing elements would imply their mechanical quantities as "hidden variables" rejected by the theorems at issue. Nonetheless, if one grants the probability density distribution (and thus, an eventual relevant quantum field, the space-time values of which are considerable as conditional "elements") as primary might be "circumnavigated" partly in a sense. That approach has a projection on Gibbs's thermodynamics: respectively, it can be considered as a generalization of Gibbs's thermodynamics after the additional admission of interactions of the states of the whole, i.e. the direct physical interactions of probability density distributions (unlike the non-interacting classical Gibbs probability distributions) and meant in the concept and quantity of quantum information.
} 
does not generate any ambiguity, contradiction or inconsistency since the usual meaning of identity or bijection is valid as to any true part of the totality (that is within the totality, properly), and the generalization, only to the totality itself after its formal definition in "mathematical transcendentalism" (Penchev 2020 August 31).

Speaking loosely, information being measured in units of bits can be interpreted as a quantity registering how many (or much) of the totality is available in a certain part of it. Once the totality has been postulated as transcendentalism does, it implies the concept and quantity of information immediately as the most fundamental one being originating directly from it. Thus, the totality being causa sui definitively generates the being universally sharing information. The being does not need any other cause than the totality, and the occurrence of the being can be described in terms of information absolutely, exhaustively, and thoroughly ${ }^{48}$.

(3) The theories of irreversible time are consistent to introducing discreteness (respectively, "leap"49) along with continuity or smoothness and this takes place explicitly in quantum mechanics and implicitly in thermodynamics. The fundamental Planck constant implies it as to the former. The phenomenological wholeness subjected by thermodynamics suggests a finite distance (or discreteness) from it, necessary for the wholeness to be investigated as such. The implicit discreteness follows the external viewpoint necessary to it and discussed in (2).

The concept of evolutionary tree, and consequently, that of abstract evolutionary tree means irreversible time. Its counterpart complementing it to reversible time can be added as a second half of a whole so that any real evolution visializable as a relevant evolutionary tree takes place only as the one half of that whole.

However, the eventual equating of abstract evolutionary tree and well-ordering (as the concept of irreversible time can be represented mathematically) means still two relations: (1) the quantity of information (for "evolutionary tree") and that of time (for well-ordering); (2) "choice" in the axiom of choice (in any branching of evolutionary tree) and "well-ordering" in the well-ordering "theorem" (in any successor of well-ordering).

"Evolutionary tree" can relate and thus unify the former members, and "well-ordering", the latter members of both (1) and (2). Thus, a more fundamental condition underlying the set-theoretical equivalence of the axiom of choice and the well-ordering "theorem" can be made clear: the "conservation of all elements in any set". As to set theory, that property seems to be so obvious that it is not formulated as an expressive axiom.

However, its corollaries as to physics and especially, to quantum mechanics are among the most fundamental laws such as energy conservation or the "conservation of energy conservation" in quantum mechanics in virtue of quantum-information conservation properly. Immediately granting conclusions of a previous work (Penchev 2020 August 17) as to the latter, one can admit that "evolutionary tree" and time as "well-ordering" may be as equated (if the system is accepted

\footnotetext{
${ }^{48}$ Particularly, quantum mechanics interpreted as a theory of quantum information is able to describe the genesis of the physical being without the theory of the "Big Bang", which continues to be unsatisfactory from a logical and metaphysical viewpoint regardless of the many experimental confirmations (which would be to be explained by any alternative theory as well).

49 "Leap" is not less consistent with reversible time as "quantum leap" and "coherent state" can demonstrate. In fact, discreteness ("leap") is added to continuity being complementary to each other in quantum mechanics, and its pair can be related to the pair of reversible and irreversible time, also complementary to each other in quantum mechanics. That is: not the discreteness by itself, but its second "dimension" to continuity is able to introduce the isomorphic complimentary second "dimension" of irreversible time to the first "dimension" of reversible time.
} 
to be conservative as after energy conservation) as subordinated (if "well-ordering" comprises only a true subclass of evolutionary trees, which can be called "conservative evolutionary trees", each of which coinciding with its "temporal projection).

On the contrary, the existence of "non-conservative evolutionary trees" would imply the occurrence of new elements, respectively new branches or spontaneous violations in well-ordering (time). For example, any self-training algorithm is a non-conservative evolutionary tree self-complicating by itself, i.e. creating new branches or adding new non-zero segments between two successive well-ordered elements. Not less, it would be able to abbreviate as branches (of an evolutionary tree) as segments (of a well-ordering).

Of course, any human being is able to do this in general. AI should be able to do this, presumably in definition. However, the present paper advocates that nature by itself would be to be able to do the same and even still in its physical level ${ }^{50}$.

That property of non-conservative evolutionary trees can explain the variability and density distribution after the set-theoretical (Dedekind) definition of finiteness, respectively, "transfinite natural number". Indeed, a self-training or self-organizing evolutionary tree possesses a variable number of elements; or vice versa: a variable set of the same power is necessarily infinite. That kind of variability can be referred also to the way for consciousness to take place "almost always", i.e. to be equated to the permanent capability of self-training or self-organization.

\section{TIME AND THE TOTALITY BY INFORMATION AND QUANTUM} INFORMATION

The viewpoint to evolutionary trees until now means the way for information, quantum information, and time to be inferred from the totality by a method coined as "scientific transcendentalism". Then, the abstract evolutionary tree can be seen as a finite projection of both information at all and quantum information.

An alternative viewpoint would suggest the converse deduction: namely starting from the abstract evolutionary trees, the class of which be generalized as quantum information, on the one hand, and as time, on the other hand: so that both generalizations result into the concept of the totality eventually under complementing conditions (even presumably more interesting for investigation).

A smooth (and thus continuous) and omnipresent medium of quantum information can be inferred as the class of all possible abstract evolutionary trees (demonstrated above). Furthermore, each of them implies the option to be well-ordered as a certain calculation of a Turing machine (respectively, as a certain Turing machine and demonstrated above as well).

In other words and again, the relation of a general class (for quantum information) and its complementing determination to just one element (for time) is what is meant. However, that class is as wider than the former as possible, achieving all able to be (whether "bodily" or "mentally"), or the "being" in the poll of philosophical reflection. Its correlative determination by time is not the least extreme, in order to be able to choose just one actual and finite existence. "Quantum information and time" can be identified as the scientific and physical (but not only) equivalent of Heidegger's Being ("Sein") and Time (“Zeit').

\footnotetext{
${ }^{50}$ All phenomena of entanglement studied by the theory of quantum information can be considered as a kind of natural non-conservative evolutionary tree non-obeying energy conservation in general, but obeying quantum-information conservation.
} 
Consequently, the proper subject of the present section can be defined as the explication of the scientific counterpart of the way for Heidegger's "fundamental ontology" to be deduced from the totality formally and rigorously, eventually under additional conditions. In other words, one need track: (1) the origin of "Sein and Zeit" from Husserl's doctrine; and (2), the latter from philosophical transcendentalism (particularly, from Kant's original one). Then, that track is to be translated from the language of non-falsifiable philosophical doctrines to that of testable scientific theories therefore abandoning the territory of philosophy provisionally and altering it with that of science, but not less provisionally:

Husserl enriched and fortified the doctrine of transcendentalism by his reductions implicitly meaning mathematics (by "eidetic reduction") as a correlative and inseparable counterpart of philosophy (by "epoché" or a corresponding interpretation of "phenomenological reduction") both unifiable by a form of sophisticated transcendentalism (as what Husserl's phenomenology can be interpreted following himself), after which "phenomenological reduction" and "transcendental reduction" are to be identified.

Another paper (Penchev 2020 October 18) considers in detail how mathematical and physical transcendentalism as a form of scientific transcendentalism is able to translate Husserl's "language of reductions" in that of the qubit Hilbert space. So, what remains is only to be demonstrated the pathway of the origin for Heidegger's fundamental ontology and his "Being and Time" from Husserl's reductions in terms of scientific transcendentalism as what the means of the qubit Hilbert space can be granted in virtue of the cited previous research.

The missing link between Husserl's reductions and Heidegger's "Being" and "Time" can be seen as the "naturalization" of the former as the latter two "existential categories". Husserl blamed Heidegger just for the "naturalization of phenomenology" 51 in "Sein und Zeit" meaning that Heidegger had restored reality in a new though sophisticated form. On the contrary, phenomenology would begin with "epoché to reality" including psychological reality or reality correlative to phenomenological psychology.

However, one can approach so: abandoning all content of "Sein und Zeit", which might be interpreted indeed as a "naturalized phenomenological psychology", only the existential categories of Being and Time to be interpreted by transcendental and phenomenological reduction correspondingly (respectively, by eidetic and psychological reduction). Then, Heidegger's "und" (in "Sein und Zeit") would mean the identity or equivalence of transcendental and phenomenological reductions proclaimed by Husserl before that. If one admits to unify Heidegger's and Husserl's pair of concepts in a single sentence, it might be: in what transcendental reduction results is Being, and in what phenomenological reduction, is Time.

However, the just cited paper of mine demonstrates the unity and resultative "naturalization" of both reductions is right the qubit Hilbert space once physical and mathematical transcendentalism has been granted. Heidegger means the same decomposition of the totality, but properly philosophically or "fundamentally and ontologically" into Being and Time.

If one explores the totality by Being and Time therefore resolving a philosophical problem inverse to that of Heidegger, they should be synthesized only by virtue of the single and unifying "totality of the totality" (or the corresponding definitive property of the totality in scientific transcendentalism), i.e. without needing any additional condition to be satisfied.

${ }^{51}$ Relevant papers as well as texts unpublished during Husserl's lifetime are contained in: Husserl; Heidegger 2011. 
If one means finite and empirical or experimental projections of Being and Time and not less, the postulate of scientific transcendentalism for the falsifiable equivalents of philosophical concepts and statements, they are to be abstract evolutionary tree and time correspondingly. The usual empirical time is definitively incomplete, partly in virtue of the time arrow implicating incompleteness. However, if one can see it from a hypothetical external ("God's") viewpoint as complete, it would represent the class of all possible abstract evolutionary trees. If one chooses a certain time, i.e. time of a certain entity, this is a certain evolutionary tree of the same entity. Thus, that entity, but not less the generalizing "abstract entity" at all, can be seen also by the option of identification of evolutionary tree and time as at all as referring to that certain entity.

What Heidegger investigates as the fundamental ontology of the totality in general and decomposable into "Being" and "Time" can be represented once again for and by science as the reality of a certain entity being thoroughly within the scope of the subject of the relevant scientific area also and in a mathematically isomorphic way decomposable into "evolutionary tree" and "time".

Any scientific entity of that kind after scientific transcendentalism means and implies invariance to the mutual transition of the viewpoint "out of the entity" and that "within the entity". Quantum mechanics defines "quantum entity" in the same way embedded just in its mathematical formalism; and vice versa as well: if one generalizes the concept of quantum entity to any entity by borrowing the qubit Hilbert space, the relevant evolutionary tree and the time of the entity at issue are to be identified (for example, as its "being").

\section{THE SEPARABLE COMPLEX (OR “QUBIT”) HILBERT SPACE INVOLVED BY QUANTUM MECHANICS}

The present section discusses: (1) how quantum mechanics involves the separable complex Hilbert space to define "entity" in its framework; (2) how the substance of that entity turns out to be quantum information after interpreting the separable complex Hilbert space as the qubit one; (3) how the concept of quantum-information substance and entities embodies the relation of Being and Time in a physical scientific, physical and mathematical way; (4) how "entity" can be defined more abstractly and philosophically generalizing the same pathway followed by quantum mechanics and information; (5) how the naïve and implicit understanding of entity in empirical experience and science can be inferred by its generalization by means of the relation of Being and Time (6) how the option of non-temporal Being (i.e. out of Time) is to be understood.

(1) Unlike classical mechanics, physics, and even science, quantum mechanics was forced to reflect on the invariance of quantum entities after the transition between the two viewpoints: internal and external to the investigated entity. This is due to involving the apparatus in the cognitive and epistemological structure of the theory.

On the contrary, the classical approach before it does not suggest that reflection in virtue of the "transparency of measurement": it does not influence the experimental results as far as that influence can be diminished under any preliminarily established threshold of exactness and thus granted as zero. Indeed, this is only a theoretical assumption meaning the absence of any fundamental restriction though the historical level of technics and technology imposes restrictions however less and less in the course of progress. Thus, the "transparent measurement" assures in classical science a trivial identity of the internal and external viewpoint to the studied entity therefore not forcing or needing any reflection. The entity at issue was accepted to be given in advance correlatively to that trivial identity. In other words, the correct research should not influence the studied entity as well as vice versa: an entity eventually influenced by the research 
means incorrectness and mistakes. Thus, constructing the entity by its research itself was unthinkable, definitively non-scientific, and maybe even anti-scientific.

This can explain one dimension of the crisis caused by quantum mechanics forced to reject the postulate of transparent measurement, due to the Planck constant and to reflect the apparent fundamental obstacle. Quantum mechanics turned out to be seemingly paradoxical: an experimental science, nonetheless definitively non-scientific or even anti-scientific according to the criteria of science and methodology before it, those of classical science and transparent measurement.

Of course, quantum mechanics is as true as "complete", the prejudice of classical science was false or "incomplete": it was not able to comprise the generalized understanding of science unconditionally necessary for quantum mechanics to establish itself. The identity of the external and internal viewpoint to the studied entity cannot be more that trivial kind of predetermined entity identical by itself to any research and referring to the implicate postulate of transparent measurement rejected and already impossible in the base of quantum mechanics forced to investigate the generalized case of an entity appearing in the course of measurement (and research as far as the measurement is to be included within the research).

The change of paradigm was so fundamental, radical and revolutionary that a great scientist as Einstein could not follow it.

However, just this new paradigm is necessary to advocate the viewpoint of the present section. One need follow the epistemological lesson and instruction of quantum mechanics and further, of quantum information in order to generalize them philosophically, in the scope of so fundamentally categories such as Being and Time.

Consequently, the problem is: What is "Entity" if be defined only by "Being" and "Time", even not only philosophically, but not less scientifically following quantum mechanics and information; (1) means the first step: to realize the lesson and instruction of quantum mechanics as a pathway to (2), (3), (4), (5), and (6).

The establishment of quantum mechanics by means of the mathematical formalism of the separable complex Hilbert space thereby unifying Heisenberg's matrix mechanics (considerable as referring to the reading of apparatus) and Schrödinger's wave mechanics (attachable to the measured quantum entity "by itself") is just what links the external and internal viewpoints, correspondingly the measured "image of the entity" on the apparatus and the entity "itself and by itself". This is not the trivial solution of "transparent measurement" in classical mechanics, physics, and science as far as the influence of the apparatus on the studied entity is not zero definitively. Anyway and though being non-trivial, this is a solution in virtue of the identification of the two mathematical interpretations of the separable complex Hilbert space: (1) as an infinitely dimensional (in general) vector space (for matrix mechanics) and (2) as the space of square-integrable functions (for wave mechanics).

However the unification of those two mathematical interpretations needs furthermore or implicitly the property of unitarity only which is able to reconcile the square-integrable functions as unitary class of vectors with each separate representative of it, i.e. a certain vector. At the same time, that unitarity is directly interpretable physically as the "conservation of energy conservation" in quantum mechanics (Penchev 2020 August 17) and more precisely, as the conservation of the measured entity energy to any possible apparatus. This proper physical consideration is to be relied not less mathematically, on the base of set theory: the logical equivalence of the axiom of choice (for the space of square-integrable functions) and the 
well-ordering theorem (for the vector space). That set-theoretical foundation of unitarity in quantum mechanics as a necessary condition to be inferred the non-trivial equivalence of the external and internal viewpoint to the entity will be utilized further as well: to be justified the solution of the philosophical problem "What is Entity in relation of Being and Time?"

(2) The result above can be interpreted also in terms of quantum mechanics properly, as the coincidence or identity of the wave function measured by the apparatus and the wave function of the entity "itself and by itself" necessary for quantum mechanics to be established as an objective science. Furthermore, any given wave function can be interpreted as a value of a new dimensionless physical free variable called quantum information and identifiable also with the separable complex Hilbert space as a whole. Quantum information can be interpreted as a generalization of the concept of information referring to infinite series or sets (Penchev 2020 June 18). A more general conservation of quantum information can be suggested (Penchev 2020 August 17) therefore admitting energy non-conservation in its framework also as an explanation of "dark matter" and "dark energy" (Penchev 2020 August 31). The properly philosophical reflection of that energy non-conservation will be utilized in (6) for justifying the option of "non-temporal Being"

Quantum mechanics is able to be reformulated thoroughly and absolutely as a theory of quantum information more relevant and more suitable for general relativity to be understood as a theory of quantum gravity in a generalized sense.

(3) The philosophical category of "Being" means a universal philosophical substance of all existing and unifying Cartesian two ones: "Mind" and "Body". They both suggest that of "Time" as far as both can exist only temporally and unlike "Space" attachable only to "Body". So, all "Being" seems to be "Time", but not "Space".

All classical physics including relativity is "bodily": both "materialistic" and "space-time " therefore keeping the Cartesian distinction of "Body" and "Mind" as absolute, and physics is related only to the former. Due to the Planck constant, the apparatus (as a hypostasis of "Mind" sharing the same property of registering empirical or experimental results) is "bodily", "material", and "non-transparent" at the same time, and not less than the studied quantum entity ("bodily" in definition as a hypostasis of "Body" sharing the same property of being registered).

So, "Body" and "Mind" turn out to be unified in quantum mechanics as the apparatus and quantum entity correspondingly, at first glance, sharing classically "Time" and its physical counterpart, the quantity of time. Soon, since Pauli presumably, the physicists have noticed that the quantum quantity of time is unique; or by his words, "it is only a number", not an operator and unlike all the rest physical quantities.

Indeed, the concept of time in quantum mechanics shares irreversibility as in thermodynamics rather than reversibility as in classical mechanics and relativity. That irreversible time of quantum mechanics, however, is inconsistent to any "coherent state" definitively reversible and implicitly meant after any physical quantity associable with a self-adjoint operator in the separable complex Hilbert space. So, quantum mechanics though stating the identity of the wave function of the measured entity with that registered by the apparatus, nonetheless, keeps the fundamental distinction between the reversible time of the coherent state of the entity "itself and by itself", on the one hand, and the irreversible time of the apparatus, on the other hand, particularly representable by the missing operator of time after Pauli.

However, this does not generate any problem in the cognitive consistency of quantum mechanics. On the contrary, the disjunctive distinction of the two kinds of time allows for 
"conserving energy conservation" 52 (Penchev 2020 August 17) in quantum mechanics as well and in virtue of unitarity of the separable complex Hilbert space originating in turn still from the equivalence of the axiom of choice and the well-ordering "theorem" in set theory.

One need complement that fundamental origin with the realization of irreversible time as the "half" of the irreversible time. Indeed, if the "first half" (of the reversible time) shares our time arrow, the "second half" should move "backward in time". Then energy conservation would be only a different way of expression for the postulate (or prejudice) of classical physic and empirical science to study definitively only what is temporal in the sense of the "first half" of reversible time and sharing our time arrow.

The same disjunctive separation of two alternative directions of time is embodied still into the mathematical formalism postulating the two twin dual spaces for any Hilbert space furthermore able to represent relevantly the concept of complementarity distributable as the relation of those two dual spaces. Indeed, the anti-isometry of them (being complex) is consistent to two opposite directions of time.

One might coin the metaphor that gravity is the same articulation of "mute difference" in physics or science at all and linking unambiguously the counterparts of the "ontological and ontic" to each other ${ }^{53}$.

Once that ontological interpretation of gravitation has been put forward, entanglement is a natural correlate of it. Gravitation in general relativity determines additionally any mechanical motion (i.e. definitively occurring in the standard direction of time) by a complementing gravitational field, in fact, representing a hypothetical motion backward again in the standard direction of time as a second fundamental component with the motion itself.

Entanglement represents the same picture equivalently, figuratively speaking, both directions of time "atemporally", i.e. as not well-ordered, but as a simultaneous set (infinite, in general) of bits, respectively elementary choices or a single qubit, respectively a choice among an actual infinite set. In other words, the equivalence of gravity and entanglement is only a physical expression of the mathematical equivalence of the axiom of choice and the well-ordering "theorem" (Penchev 2020 August 30; 2020 August 31).

The same problem of dividing a whole into two halves and eventually interpreted as the two directions of time is resolved in three different ways in: (a) the "classical" quantum mechanics (from which the Standard model originates); (b) general relativity; (c) the theory of quantum information able to unify the first two ones as a nonstandard ${ }^{54}$ form of quantum gravity:

\footnotetext{
${ }^{52}$ By the way, Pauli's understanding of time as "only a number" was linked closely to energy conservation in quantum mechanics, by which he could forecast the existence of neutrinos as necessary, right for the conservation of energy. Indeed, an operator associable with time would imply energy non-conservation in general and just as the BKS theory suggested (Bohr, Kramers, Slaters 1924).

${ }^{53}$ Indeed, the physical theory of gravity means the attraction of existing rather than "being" (ontological) entities. However, the metaphor means rather the linking of the space-time and energy-momentum tensors in each point, by which the gravitational force field acting in the same point can be defined.

${ }^{54}$ It can be called "nonstandard" due to the following. If the standard approach is defined by "secondary quantization" of gravity, and thus general relativity obeys quantum mechanics, the nonstandard quantum gravity meant here considers general relativity and quantum mechanics as two complementary theories sharing the same subject. Particularly, gravity (as it is defined implicitly and contextually in general relativity) cannot be still one interaction addable to the rest three ones in an eventual, cleverly extended the Standard model, but an "equal partner" to all the three meaning the same: gravity in a smooth or continuous manner, and the complex interaction consisting of the three elementary ones meant in the Standard model in a discrete (quantum) way.
} 
(a) The consideration as to the entity by itself is "atemporal", or from the viewpoint of the axiom of choice, but nonetheless, "temporal" as to the results of measuring the same entity. Then, the whole is divided into two "temporal halves" meaning actually and fundamentally only one of them. For example, the so-called Copenhagen interpretation prohibits any questions as non-scientific about the other half of the same entity simultaneously with the first one being measured really. However, the mathematical formalism itself allows for the interpretation of the "other half" as the implicit dual "twin" of the Hilbert space to which the measurement at issue refers. It is anti-isometric, and the property of anti-isometry can be interpreted as relevant to the reversed time arrow.

(b) The way of dividing the whole into two halves is controlled in general relativity again by the equivalence of the axiom of choice and well-ordering "theorem", respectively the equivalence of choice and well-ordering. Anyway, the formal and mathematical description of that control is essentially different in general relativity in comparison with quantum mechanics therefore making an impression of being even incommensurable or inconsistent to each other. One need realize the approach of general relativity in detail in order to be able to compare it with that of quantum mechanics in (1) above:

The temporal and atemporal considerations in turn constitute a pair being only disjunctively distinguishable in quantum mechanics: the former belongs to the measured results and readings attachable to the apparatus properly; the latter corresponds to the quantum entity itself and by itself, i.e. before measurement. The identity of wave function able to describe both in virtue of the unification of matrix mechanics and wave mechanics links them as the same therefore satisfying the fundamental epistemological criterion of objective cognition.

The complimentary option would suggest for the temporal and atemporal considerations not to be divided alternatively due to the axiom of choice, but ordered in virtue of the well-ordering "theorem", and thus simultaneous in a sense, i.e. as if in a "meta-time", however necessarily coinciding with "time" in physics because of the unifying ability of the totality.

That well-ordering of the temporal and atemporal considerations is realized by the pair concepts of a function and its derivative: as in general relativity as in classical mechanics and physics therefore needing for the relevant manifold to be smooth.

Indeed, any function interpreted as the representation of a physical process is temporal in classical physics. Though its derivative as a different function (in general) can be interpreted as another physical process and thus temporal, anyway it is a temporal in relation to the function itself, to which it is derivative, in the following sense: it refers to two different values of it in different moments of time simultaneously, i.e. as a single one definitively.

Consequently, classical physics and general relativity unify the temporal consideration by a relevant function with its atemporal counterpart by its derivative unambiguously inferable from the kind of the function at issue. So, the Lagrangian formulation of any physical process unifies its temporal and atemporal considerations as a fundamental condition. Nonetheless, the Hamiltonian description of the same process is possible always (especially intuitively justifiable in quantum mechanics due to the Planck constant) realizing the disjunctive division of the temporal consideration from the atemporal one.

The concept of "curving" featuring pseudo-Riemannian space can make visible the synthesis of temporality and atemporality in general relativity by both irreversibility and reversibility of time. Time in general relativity as in classical mechanics is reversible, but it is "curved" unlike it. The curving of time implies a time derivative of time (or a meta-time derivative of time, but 
"time" and "meta-time" are to be identified due to the totality), and therefore the dimensionless and thus physical quantity of information (respectively, entropy) can be interpreted as it. So, that time derivative of time realizes the unification of temporality (for the time itself) and atemporality (for its time derivative) as to general relativity and vice versa as well: the whole in it consists of temporality (i.e. the time itself) and atemporality meant by the curving of the time (i.e. the time derivative of time or information in the final analysis therefore allowing for its unification with quantum mechanics as identifying gravity and entanglement as follows).

(c) One can juxtapose the two theories themselves, quantum mechanics meant in (a) and general relativity, in (b), considered successively, now simultaneously therefore realizing once again the equivalence of choice and well-ordering, reconfirmed by the corresponding axiom and "theorem" in set theory as to infinite sets. The successive consideration of them furthermore being usual as to the scientific "common sense" suggests for them to be different theories according to the following clearly distinguishable and essential features.

Quantum mechanics means additionally discrete physical actions for the microscopic quantum entities together with continuous actions of the macroscopic apparatus describable only by classical mechanics. The physical interactions accessible to quantum mechanics are only three, weak, electromagnetic, and strong and represented exhaustively by the Standard model. What is entanglement studied by quantum information or how it is to be related to those three interactions are big unsolvable and unarticulated yet problems.

General relativity means continuous (even smooth, ordinarily) and macroscopic actions and a single interaction, gravitational and absolutely different from the mentioned three ones of the Standard model.

The scientific common sense does not see any other option about their relation than the suggestion for the continuity (smoothness) of classical mechanics, special and general relativity to be only seeming and approximate, in fact, due to a huge difference, even in decades of exponents, between the microscopic actions of quantum mechanics and the macroscopic ones studied by the previous theories: a difference predetermined by the magnitude of the Planck constant. So, nature should be fundamentally and universally quantum, i.e. discrete, but the relevant granular structure of the world is so fine that it seems to be continuous or smooth, inaccessible even to experiments because of Heisenberg's uncertainty rather than only as to our empirical experience.

Though obvious, that approach meets at least two insurmountable obstacles:

(a) The granular structure implies "hidden variables" referring to any single "grain" (e.g. as the Boltzmann statistic thermodynamics infers the phenomenological and thermodynamic quantities directly from the complemented mechanical quantities of relevant "grains" such atoms, molecules, etc. and developed in De Broglie and Bohm's theory as to quantum mechanics). However, the formalism of quantum mechanics excludes fundamentally the option for them to exist in virtue of the "no hidden variables" theorems (Neumann 1932; Kochen and Specker 1967).

(b) The fundamental and universal quantumness of the world implies "quantum gravity": a granular analogue underlying the macroscopic phenomena of gravity described by general relativity just as the Maxwell equations of electromagnetism can be inferred as an approximation of quantum electrodynamics. The strong and weak interactions are specifically quantum, i.e. they do not possess any macroscopic analogues. The electromagnetic interaction is not specifically quantum, i.e. it possesses a macroscopic analogue. However, the fundamental and universal 
quantumness excludes the fundamental option of only macroscopic interactions to which gravity might be enumerated. Then, quantum gravity sharing a base similar to quantum electrodynamics seems to only be admissible logically. Nonetheless, a relevant theory of quantum gravity has not been created yet regardless of a century of huge efforts. The experimental confirmations of scientific hypotheses, eventual applicants for that, are much weaker than those of general relativity. Moreover, there are proofs that the usual formalism of quantum mechanics (by the separable complex Hilbert space) and the conjecture of quantum gravity are inconsistent to each other.

One can notice that both obstacles would be surmountable if the separable complex Hilbert space might be replaced as the mathematical formalism of quantum mechanics by another suitable one e.g. by the Broglie - Bohm interpretation of quantum mechanics by "local hidden variables":

Competitive models are verifiable by relevant experiments to phenomena which only one of them implies as observable. According to falsificationism, the one rival is to be removed, but the other one only continues in the competition without winning ultimately.

All the phenomena of entanglement experimentally are confirmed well enough (i.e. not meaning extremely improbable "backdoors" of alternative explanations furthermore ad hoc and each of them applicable only to a single kind of experiments). They remove any hypotheses of local hidden variables seeming to be necessary for both (a) and (b) therefore reconfirming their status of being crucial obstacles preventing the conjecture of the scientific common sense about the fundamental and universal quantumness of the world, but in favor of quantum information.

However, all experimentally corroborated phenomena of entanglement as well as their theoretical reflection by quantum information violate the symmetry of mutually complementary choice as in (c), on the one hand, and their successive ordering as in "(b) after (a)" as to the pair of quantum mechanics and general relativity therefore calling for a relevant explanation; it is due to the postulate of the totality, or the following in detail:

The aforementioned symmetry is a corollary from the equivalence of the axiom of choice and the well-ordering "theorem" fundamental of set theory. However, it is to be interpreted as still one implementation of the equivalence at issue rather than its rejection. The peculiarity is due to referring to a special and unique, properly total entity what the totality is: it is postulated to be the first (respectively, last, after the inverse ordering) element of any possible ordering, even more, in an absolute sense. Therefore, the implementation of that equivalence to it, meaning that the one "twin", namely that of the well-ordering theorem, is presupposed by the postulate of the totality implying as ordering as a first (last) element in it, and thus well-ordering totally, needs the second "twin" to be relevant to "choice" in the axiom of choice ${ }^{55}$ :

\footnotetext{
${ }^{55}$ One can demonstrate the same peculiarity abstractly, by the special kind of choice to the extremal (i.e. whether the first or the last) element of well-ordering in comparison with the choice of any other element of the same well-ordering. The latter admits for any element two local choices: the element immediate successor versus the element immediate ancestor, and after that, divides all elements into two well-ordered classes, in the one of which it is the first element, and in the other of which it is the last element. Thus, well-ordering and ordering are indistinguishable locally in relation to it. They are distinguishable locally only by the extremal element, to which the local choice is initially and fundamentally global since it predetermines disjunctively the global kind of ordering: either "greater" (">") or "less" ("<"). If the element at issue is the extremal one, the two classes being simultaneously possible in the former case turn out to be complementary to each other being impossible simultaneously. And vice versa as well: if complementarity in that rigorous, formal and mathematical meaning is available, this implies the totality
} 
That second "twin" is just the complementarity of general relativity and quantum mechanics representing the same: the totality seen from a physical viewpoint in two complimentary ways; for example, externally (as if out of the totality) as to quantum mechanics versus internally (as if within the totality) as to general relativity. Thus, the symmetry of choice and ordering is violated only seemingly. In fact, its occurrence as a special case of an extremal element (what the totality is, definitively) implying only the complementarity of general relativity and quantum mechanics.

That consideration implies for quantum mechanics to be reformulable without the postulate of the totality, i.e. without Bohr's complementarity therefore admitting the phenomena of entanglement, and even without it, very well confirmed experimentally.

That option can be represented furthermore formally and mathematically as follows. Any well-ordering can be transformed into ordering by the simultaneous consideration of its two complimentary well-orderings ${ }^{56}$. The one "half" consisting in the one well-ordering is situated thoroughly either before or after the other "half" consisting in the complimentary well-ordering so that the totality is simultaneously the least element of the one well-ordering, but the greatest element of the other well-ordering.

So, if one utilizes the "classical" quantum mechanics after Bohr's complementarity or the so-called Copenhagen interpretation, including the generalization of the Standard model as well, only one single quantum system (and its Hilbert space) is meant and the two well-orderings possible only as complimentary are implemented by the anti-isometry of the two dual Hilbert spaces to each other.

However, if one uses the quantum-information reformulation of quantum mechanics relevant to entanglement, the description of the "classical" quantum mechanics can be kept as to the system being a whole or as to any single quantum system being entangled with others therefore keeping implicitly as a single well-ordering as the concept of the totality relevant to the quantum system of the totality. Though the concept of quantum information is applicable (and thus unifying) to both "classical" quantum mechanics and entanglement description, the entanglement by itself needs more than one qubit Hilbert space for being investigated therefore referring to a series of "totalities", at least one and its two complementary well-orderings implied by it. This is due still to the definition of information able to identify alternatives even in virtue of its unit, "bit" to be representable as an elementary choice between two equally probable alternatives, thus as minimal as possible.

Being inherently twofold and referable as to a single qubit Hilbert space as to many entangled ones, quantum information allows for transferring the worldview of quantum mechanics and general relativity complementary to each other (as in the previous paragraphs of "c") into that of special kind of "quantum gravity": not only identifying it with entanglement, on the one hand, but furthermore and extremely unexpectedly with gravity described by general relativity. Consequently, general relativity "as it is" turns out to be a theory of quantum gravity simultaneously and not less ${ }^{57}$.

being the first element in the one complimentary well-ordering, but the last one in the other complimentary ordering; thus, the totality can be defined even once again formally as a disjunctive relation of ordering and well-ordering by the property of complementarity relevant only to the latter. Further, as Bohr's complementarity as the discussed complementarity imply the just defined rigorous meaning, and thus the postulate of the totality in the final analysis.

${ }^{56}$ Please mean the previous footnote.

57 The worldview relevant of that unification of general relativity and quantum gravity includes two independent components: (1) the cyclic universe in Giordano Bruno's or Nicolaus Cusanus's manner: "the 
Now, one can discuss how the theory of quantum information resolves the fundamental problem at issue, namely that "about the half of any whole", as to the case (c). The main novelty is the fundamental contextuality or inseparability of the whole after which the halves can be as non-entangled as arbitrarily entangled. All cases can be unified by the concept of information whether classical or quantum. The case of a single qubit Hilbert space studied by the "classical" quantum mechanics corresponds to the whole, respectively to the totality, and the case of entangled qubit Hilbert spaces investigated by the theory of quantum information means to "halves" linked inseparably. Furthermore, the so-called "classical" quantum mechanics can be reformulated thoroughly and absolutely in terms of quantum information. Thus, quantum information turns out to be the relevant generalization of quantum mechanics including the problem "about the half of any whole". A bonus to it is the option of identifying gravity according to general relativity with quantum gravity.

The intention of the present subsection is to prepare a fundamental and ontological, but not less scientific generalization of "Being" as quantum information and "Time" as the physical quantity of time or well-ordering. The objectivity is the definition, in terms again of both fundamental ontology and quantum information, as of "Entity" as of "physical or mental entity". It will be realized in detail in the next subsection. "Entity" whether fundamentally and ontologically or scientifically means a structure isomorphic to a bit of information: "Being" as the totality (respectively and scientifically, any whole) and only a "half" of it as "Time". In other words, "Entity" should be defined just as the "temporal half of any whole". Thus, using "Entity" or "entity", one means the temporal (or well-ordering) aspect of the "problem about the half of any whole" (including the totality as a whole being absolute definitively).

(4) "Entity" can be defined more abstractly and philosophically generalizing the same pathway followed by quantum mechanics and information. One needs that understanding of an entity able to oppose restricting it only within the material hemisphere of the world as the philosophical "Object" or as many "objects" studied by modern science by a definitively external observer, or the philosophical "Subject" therefore following Cartesian dualism unconditionally. Speaking loosely, the intended definition of "Entity" (or respectively, many scientific entities which cannot be the "objects" for the research of classical science) is to be both "subjective" and "objective", but nonetheless, neither "subjective" nor "objective".

One may also use the metaphor or even rather the example of the classical distinction of "subjective probability" and "objective probability" able to share an identical axiomatic base. This fact can be interpreted philosophically so: the concept of probability (or its qualitative counterpart of possibility) can be relevant to the intended unification of "Subject" and "Object". Indeed, information or quantum information can be understood as a relation (and quantitatively, ratio) of probabilities, namely those of the alternatives of that choice or many choices to which information refers. Furthermore, information and quantum information are associated with the fundamental and ontological "Being" transferring it into the framework of falsifiable scientific theories (such as the theory of information or quantum information, in turn equivalent to quantum mechanics).

Though unifying "Subject" and "Object", "Being" and "Time" are opposed to each other anyway. Particularly, being restricted to be valid as to the "half" of "Being" ("Being" as the

universe in a single quantum"; (2) the identification of global and local space as in the Standard model or gauge theories, but identifying as those of the pseudo-Riemannian space of general relativity and the qubit Hilbert space correspondingly. The relevant worldview is discussed in detail in: Penchev 2020 August 31. 
totality and thus, as a whole), "Time" it is able to generate the material hemisphere of "Being" if it is postulated to be therefore implying energy conservation (respectively, matter conservation) as the essence of that hemisphere. On the other hand, energy, matter, and their conservation can be seen by the availability of the other "half" of "Being" (i.e. time running backwards) as a permanent and conserving whole in the former, "first half" of "Being" (i.e. time running normally, "forwards").

Noticing that both halves of "Being" need be divided disjunctively if the material hemisphere is the case, classical information rather than quantum information (after which material and mental hemisphere are indistinguishable from each other) is relevant to it. Moreover, classical information can be granted as definitive to the material hemisphere from the viewpoint of the theory of information, in the framework of which "entity" (for "Entity") is to be defined as the class of all equations able to refer to the fundamental equation of quantum information (for "Being") and classical information (for "Time") ${ }^{58}$, respectively the "ratio" of a qubit and the qubit to which the Planck constant relates presumably.

The same fundamental equation can be interpreted also as a quantitative expression of the relation of "infinity" and "finiteness", whether in philosophy or in mathematics, as far as quantum information reflects the information of infinite series or sets unlike classical one, to finite ones.

(5) The naïve and implicit understanding of entities in empirical experience and science can be inferred from its generalization by means of the above understanding of the fundamental and ontological Entity as the relation of "Being" and "Time". That naïve representation of entity is correlative to the scientific dualism, after which any entity is to be situated within the "material hemisphere": so, any science referring to the "mental hemisphere" such as psychology is unable to define "entity" in a way both relevant and consistent in the framework of the cognitive episteme of Modernity (articulated first in Descartes's dualism).

Anyway, that naïve "entity" can be inferred from the relation of "Being" and "Time" as above as a particular case, i.e. adding a relevant condition consisting in the following: only the projection of the relation of "Being" and "Time" into "Time" is to be meant as that idea of entity relevant to science and empirical experience. Excluding general relativity (if one has enumerated it among the modern episteme), the scientific and empirical "time" is meant as running evenly and only "forwards", i.e. to be Newtonian. Energy and matter conservation (unified after special relativity) being the natural correlate to that Newtonian time can define "entity" physically as constancy in time and underlying any other entity therefore being physical scientifically or material philosophically in the final analysis or in the most fundamental level.

Thus, both "transparent measurement" and "bodiless observer" of classical physics and science are only another expression of the material (or physical) constancy in the (Newtonian)

\footnotetext{
${ }^{58}$ The Schrödinger equation fundamental for quantum mechanics belongs to that class. It equates the first time derivative of wave function (referable to classical information) to the second space derivative of wave function (referable quantum information) up to one spatial function interpretable as potential field. A previous paper (Penchev 2020 June 18) contains a few relevant ideas, but the rigorous justification is postponed for a future study. One can discuss whether the Einstein field equation fundamental for general relativity belongs to the same class. It seems to describe rather the links of both "halves" as projected on the "temporal half". Therefore, it means quantum information as transformed into classical information in advance as a premise and only after that projected on the chosen alternative of time running "correctly", i.e. "forward". In other words, the viewpoint of the physical theory of information is able to show that both equations express the same, but in different "languages".
} 
time of the modern episteme. One may involve the metaphor of an imaginary quantity relevant to both "transparent measurement" and "bodiless observer" and being a "Newtonian time derivative of the physical constancy" (or stability).

However, if the cognitive analogue and generalization of general relativity (abandoned the Newtonian time and therefore both "transparent measurement" and "bodiless observer" substituted by the measurement and observer's reference frame only to which that of the studied entity can be determined unambiguously) is to be introduced after that, a "mental reference frame" of the researcher whether an individual or a group only to which the cognized entity is definable univocally would correspond. Following literally the analogy, that "mental reference frame" should be thought as a second material image of the entity at issue, but in the other "temporal half" i.e. in time running "backwards". Once again: "mental reference frame" would be a material reference frame after reversing the time arrow. Then, general relativity would share the same formalism as a "mathematical epistemology" (or "mathematical theory of cognition") inexistent yet.

The analogy can be followed also in a generalized sense if one means the equivalence of entanglement and gravity, or general relativity as quantum gravity by the method sketched above. Instead of a second material reference frame moving backwards in time as "mental reference frame", a coherent and therefore temporally reversible "superposition of mental entity and material entity" is determined additionally by their entanglement presumably corresponding to the cognitive episteme in a certain age to a class of equivalence meaning all possible theories of an entity or subject possible in the epoch in question. That generalized analogy would generate another "mathematical epistemology" (or "mathematical theory of cognition"), but equivalent to the former.

(6) The "ridiculous" option of non-temporal "Being" (i.e. out of "Time") is to be understood as well as "Entity" should be generalized relevantly. One more step should be taken following the direction marked in series of the stages (a), (b), and (c) in Subsection (3) of the present section above:

The fundamental and ontological Entity was defined there by the relation of Being and Time, or a projection of "Being" on "Time", or that of the whole to the only one temporal "half" of it, in fact, regardless of which of both. However, the half chosen by the projection is running forwards definitively (respectively, the unselected one refers to time moving backwards and unobservable empirically or experimentally directly).

That projection can be measured in the units of bits or qubits therefore representing classical or quantum information: what the mathematical and physical essence of "Being" is. Though those projections being entities are information whether classical or quantum, the latter admits in addition the case of generalized projections not decomposable to any whole consisting of two orthogonal dimensions or alternatives (such as those in a bit of information): that is arbitrarily "entangled" alternatives. In other words, the generalized entities contain a nonzero coherent part (not necessary to be a true part) of reversible time therefore excluding for the time projection to be determinable unambiguously and rejecting to be any quantity of classical information.

Those generalized, entangled entities being essentially atemporal whether only partly or thoroughly can be visible on the temporal "screen" (e.g. that after general relativity) only as "dark shadows" (e.g. as dark matter and dark energy). Two metaphors, even quite different from each other, might be relevant: (a) by Plato's "cave"; (2) by a processing of a computer, being visible only partly as moving images on its screen. 
(a) Those "atemporal entities" need additional dimensions in comparison with those of the "wall" only accessible to the observation of the "chained people". Whatever be in themselves, they can be seen on the wall only as dark shadows without any structure unlike the wall itself, which is visible in detail, e.g. together with its relief and irregularities, colored unevenly therefore possessing a quite clear structure and texture. Nonetheless, the chained might suggest that what is visible only as "dark shadows" possesses by itself a certain structure though absolutely unknown and presumably being absolutely different from the texture of the screen. "What might that structure be?" will be articulated below as a reasonable problem for future research.

(b) The work of any computer results (as a rule) on its screen therefore, but contains a huge part of incomplete processing absolutely necessary for the ultimate results on the screen only visible for us. One can figure that the case is a movie projected on the screen therefore sharing the "time of the plot". All incomplete processing necessary for the movie to appear on the screen does not occur in the time of the plot and thus it is atemporal to it.

So, one can imagine the universe as an immense quantum computer with hidden incomplete processing but visible on its temporal screen (with time referring only to the "plot of progress" of all existent therefore starting from the "Big Bang"59). Thus, the meta-time of processing will be defined to be hidden in relation to the "time of the plot" processes (i.e. the atemporal entities in question) as computational. The structure of "dark shades" turns out to be quantum and algorithmic, the incomplete processing of reality before it to be able to appear of the "temporal screen" of our experience and not yet "prêt-à-porter" (better, "prêt-à-regarder" or "prêt-à-observer")

All the classes of calculations in quantum computers are operators in the separable complex Hilbert space (respectively, in the qubit Hilbert space). What is visible on the temporal "screen" are all physical quantities as they are defined in quantum mechanics; that is: the Hermitian (self-adjoint) operators. All other operators which are neither Hermitian nor unitary and therefore do not conserve energy are visible only by their partial projections being Hermitian and conserving energy. Just those non-Hermitian operators are "incomplete calculations" visible only as "dark shadows" by their projections being Hermitian operators.

The complete calculations visible as physical quantities are visible only outside of the universal quantum computer (what the universe is) being situated on its "screen" following the metaphor in (b). They suggest for the "computer" to be a whole and as far as that whole is the universe itself, the relevant whole is absolute, i.e. the totality. The corresponding Hilbert space whether qubit or separable and complex is a single one not involving neither phenomena of entanglement nor any Non-Hermitian operators. It is very well studied by the "classical" quantum mechanics culminating in the Standard model.

However, we know that $95-96 \%$ of all the universal matter and energy are "dark", that is only "dark shadows" of incomplete calculations invisible by themselves on the temporal "screen of the computer" otherwise than as just those "dark shadows" without any structure in principle.

Their structure is visible only within, inside of the "computer" and it is that of certain incomplete calculations, or physically, various phenomena of entanglement. The definition of entanglement implies the involvement within the computer rather than its contemplation outside, i.e. by an "external observer watching the "temporal screen" just outside. After entanglement, e.g.

${ }^{59}$ The question about what had happened "before the Big Bang" is meaningless, or speaks one jokingly, it might mean only the "titles of the film" representing the names of its creators (that is God's team), which can be "before the Big Bang". 
the one quantum system "observes" another quantum system inside of it partly (according to the degree of entanglement: the more is entanglement, the greater is the part inside of the observed system).

So, the process of de-coherence, in which the degree of entanglement decreases gradually, can be interpreted as the advance in calculation: if there is no entanglement (there is zero entanglement), the calculation is complete, and the result is ultimate. It appears on the "temporal screen" visible in its structure rather than only as an amorphous "dark shadow" for the external observer.

The atemporal entities being non-ultimate or as if in the process of being created can be reflected fundamentally and ontologically just as the discussed "ridiculous" option of non-temporal "Entity": only after its ultimate creation as if out of "Time", but within "Being", it is able to fall into "Time" already absolutely structured, but aging and therefore destined to "Death".

X STILL ONE APPENDIX: ENTANGLED EVOLUTIONARY TREES AND THEIR TEMPORAL CORRELATES ("TIMES”)

If evolutionary trees can be considered as a finite image or projection of wave function, entangled evolutionary trees would correspond to the option of entangled wave functions. Analogically, they can be thought as non-ultimate evolutionary trees being in process of creation visible only partly as vague intermediate items of the evolutionary tree at issue and flickering fleetingly and for a short time. If "the normal" ultimate evolutionary tree corresponds to a single relevant time for its development, the entangled transitional evolutionary tree suggests more than one time therefore competing with each other, but reduced to the single time of the ultimate result.

Thus, the entangled evolutionary trees can be considered also as incomplete calculations of the really observable one appearing only as interim fleeting items, difficulty provable and rather missing in the ultimate evolutionary tree.

The entangled evolutionary tree in relation to the non-entangled (i.e. "normal") evolutionary tree can be defined as containing at least one convergence: the latter contains only bifurcations without any convergence. Thus, the "normal" evolutionary tree corresponds to a single time relevant as to any branch as to all of them: then, isosynchronous lines (correspondences) exist between all branches allowing for the well-ordered chronology of all items regardless of the branch to which each of them belongs (e.g. one can state that a certain insect had appeared before a certain mammal though they belong to absolutely different branches of biological evolution).

On the contrary, any entangled evolutionary tree contains at least one segment starting by a bifurcation and ending at a convergence, within which time is not determined unambiguously: there are at least two times in that segment. Anyway, it can be decomposed into the corresponding number of normal evolutionary trees following a simple and obvious method: the subtree after convergence be divided as identical copies after each of all converging branches within the segment in question.

As far as the converging branches are distinguishable by different probabilities (definable e.g. by the number of individuals sharing the same converging branches), many of them would not 
remain any notable tracks. The absence of any convergence in the normal evolutionary tree allows for only conventional or conditional definition of branch probability ${ }^{60}$.

The normal evolutionary tree implies a corresponding single well-ordered time (though the tree itself is not ordered well) since any branch is well ordered. On the contrary, the entangled evolutionary tree needs more than one time for the segments before any convergence to be restorable well-ordering in any branch (as after represented by a few normal evolutionary trees, i.e. more than one).

The sense of entangled evolutionary tree as incomplete calculations can be visualized by many calculations in parallel (in any entangled segment) anyway separable into calculations in series of a usual Turing machine; that is: being in parallel yet, the calculation of the corresponding relevant Turing machine cannot finish as far as it needs them to be ordered in series, or in other words, to be representable on the "temporal screen", only on which Turing machine can process and processes information.

\section{THE QUBIT HILBERT SPACE GENERALIZED TO TEMPORALITY AT ALL}

The qubit Hilbert space is a method of representing the separable complex Hilbert space in a way more suitable to emphasize the information structure of it, and especially, both distinctiveness (disjunctivity) and identity (thus together, complementarity) of well-ordering and choice: respectively, the axiom of choice and the well-ordering principle. The two complementary aspects are distributed disjunctively in the global space consisting of all qubits as a well-ordered series of units, on the one hand, and local space within any qubit representing a simultaneous choice between an infinite series of alternatives, on the other hand.

Thus, temporality at all, or the philosophical "Time" as well as the philosophical "Entity" are embedded in the qubit Hilbert space itself: "Time", in the global space; and "Entity", in the identity of local and global space ${ }^{61}$. Though originating from quantum mechanics, the qubit Hilbert space is a formalism relevant to the philosophical "Time", temporality at all, and particularly, to the abstract evolutionary tree or to any exemplification of it.

The qubit Hilbert space is defined to be determined unambiguously to any separable complex Hilbert space utilizing the property of any two successive "axes" of the latter to be two orthogonal space of the same Hilbert space therefore able to constitute a qubit. That is: any separable complex Hilbert space consisting of " $n$ " axes corresponds unambiguously to a qubit Hilbert space consisting of " $n-1$ " qubits. If any of both is infinitely dimensional, the other one is infinitely dimensional, too.

If the separable complex Hilbert space is one-dimensional, i.e. isomorphic to the field of complex numbers, no qubit Hilbert space corresponds to it. In other words, "qubit" can be defined as a relation of two fields of complex numbers, respectively, a sub-algebra of quaternions defined by the algebra of quaternions and the additional one-dimensional relation specifying the

${ }^{60}$ For example, objective probabilities of branches can be defined after bifurcation, and subjective probabilities, before convergence. Entangled branches need quantum probabilities definable by the coincidence of subjective and objective probability, and thus, both bifurcation and convergence of the same branches. The dis-entanglement to normal evolutionary trees (i.e. to only objective probabilities) can be expressed as a corresponding multiplication by the number of conventional subjects for each branch of subjective probability.

${ }^{61}$ By the way, the identity of local and global space as the separable complex Hilbert space is borrowed from the Standard model therefore sharing the same implicit definition of "Entity" though exemplified as to "physical entity" in its framework. 
relation of the two fields of complex numbers. Thus being three-dimensional resultantly, that sub-algebra is isomorphic to a "flat" three-dimensional vector space what the usual Euclidean space of geometry, physics, and our experience is.

Consequently, still the mathematical structure of the qubit Hilbert space makes visible and obvious the implicit link of the separable complex Hilbert space and the three-dimensional Euclidean space of our experience, experiments or readings of any apparatuses. If one means the "classical" quantum mechanics, after which the conserving energy is able to divide the studied quantum entity and the apparatus by the abyss of decades of exponents in the magnitude of energy, the implicit link of the empirical and theoretical spaces is much more relevant. However, if one abandons the "classical" glasses of energy conservation substituting it by quantum-information conservation, the relevant approach is the explicit link between them after the qubit Hilbert space.

Furthermore, the qubit Hilbert space unlike the separable complex Hilbert space is able to represent much better the case of any entanglement being a relation of "rotated" separable complex Hilbert spaces therefore not being representable by a single separable complex Hilbert space $^{62}$. For example, any qubit of the qubit Hilbert space can be interpreted as the relation of the successive "axes" of the same qubit Hilbert space as the relation of axes (including "number-sake") of two different qubit Hilbert spaces (therefore representing their "rotation" to each other).

\section{TEMPORALITY AND (QUANTUM) INFORMATION}

Quantum mechanics reformulated as the theory of quantum information as well as the "classical" quantum mechanics culminating into the Standard model suggest a generalized approach to what "quantum entity" is, based on the relation of "coherent state" and "temporality" after the fundamental mediation of measurement. On the one hand, that approach can be reduced to the particular case of "transparent measurement", shared by classical physics and science; and on other the hand, it can be generalized in turn and transferred in the pole of the non-falsifiable philosophical reflection after the fundamental "Being" and "time" allowing for a new, innovative, and heuristic understanding of "Entity" in philosophy.

The latter suggests that the approach of both temporality and quantum information, particularly embedded in the mathematical formalism of the qubit Hilbert space can be returned back: from the non-falsifiable pole of philosophical reflection to the much more restricted region of scientific methodology thus falsifiable, at least partly, distinguishing sciences into two large groups: those in which it is applicable, heuristic and fruitful; and those in which this is much or more invalid.

General relativity, which creates or utilizes an absolutely different (at first glance) mathematical formalism, namely that of pseudo-Riemannian space $^{63}$, can be reinterpreted as a

\footnotetext{
${ }^{62}$ Due to infinite dimensionality, it is able to be extended unlimitedly therefore being able to unify any number of separable complex Hilbert spaces, but under the necessary condition not to be "rotated" to each other.

${ }^{63}$ The pseudo-Riemannian space of general relativity and the separable complex Hilbert space (from which the qubit Hilbert space originates) can be opposed in a few conceptual "axes": dimensionality, curvature, and convexity. Indeed, the former is real (a vector space on the field of real numbers), the finite- (four-) dimensional, arbitrarily "curved" in any point, and non-convex (due to the imaginary time dimension); on the contrary, the latter is complex (a vector space of the field of complex numbers) infinite-dimensional, constantly "flat" and convex (though complex). Thus, but only at first glance, those
} 
theory of quantum gravity by the crucial mediation of both fundamental concepts of temporality and quantum information (as it is elucidated in detail above). Thus, general relativity falls as the first and exceptionally successive example for the methodology at issue to be implicitly applied (or applicable) even before being articulated as "natural cybernetics of time" (as here) or otherwise.

That methodology possesses at least two essential aspects: (1) to be an interpretation of the qubit Hilbert space in terms and notions of a certain scientific area; (2) to resolve the problem about the "half of a whole" as to that area after applying the qubit Hilbert space.

A philosophical reflection as well as a formal and mathematical generalization of that methodology is called "natural cybernetics of time". It is called to elucidate how temporality is involved in the investigated special area in fundamental way: namely to control it after conserving quantum information (which can be interpreted as a generalization of the transmission of information as to the "classical" cybernetics of Norbert Wiener) so that any whole is just a stable whole in the course of time.

As the initial ideal of cybernetics, it is able to unify quite different scientific domains demonstrating them as different interpretations of the same mathematical structure as what the qubit Hulbert space turns out to be postulated in the final analysis.

\section{CYBERNETICS AND ITS CONTEMPORARY REFORMULATION IN TERMS OF QUANTUM INFORMATION \\ Cybernetics appeared after the postulation of general laws of control due to transmission and processing of information. Biological and technical systems, and subsequently many others, can be identified from the viewpoint of cybernetics sharing the same formal and mathematical structure.}

The essential features of cybernetics seen from the viewpoint of quantum information are: (1) the base of information, which is a dimensionless, fundamental physical and mathematical quantity furthermore able to unify the foundations of physics and mathematics, but being independent of the material implementation of the system: biological, technical, social or whatever else; (2) the initially emphasized similarity of technical and biological systems is rather accidental, and the essence of which is the mathematically isomorphic description of whatever system with circulating whatever information (3) information is to be generalized in way to include quantum information as the information of infinite sets and series and thus: the idea of control penetrates even in physical and mathematical (i.e. represented formally and abstractly) systems; (4) information is understood as a relation of choice and well-ordering, and particularly as relevant to the equivalence of the axiom of choice and the well-ordering theorem in set theory; (5) the idea of feedback (respectively, coefficient of transmission) especially negative and thus stabilizing is temporalized by the relation of reversible and irreversible time as well as two irreversible times, "forwards" and "backwards", different, even "curved" courses of time ${ }^{64}$.

spaces seem to be unable for unification. Nonetheless, all three enumerated opposed peculiarities being together can be balanced absolutely in exact equivalence. Moreover, that equivalence is underlain by a single mathematical structure relevant to the concept of quantum information (Penchev 2020 August 17).

${ }^{64}$ For example, the problem about the "half of the whole" of a system with feedback seems to be the following. One is to divide the transmitting coefficient or function of the system as a whole, i.e. as a "black box" into two preferably disjunctive parts, one of which is represented by the coefficient or function of feedback (i.e. the half of the process of transmission running backward). Then, "entity" would be determined unambiguously by a relevant kind of mathematical relation (for example, ratio) between the 
Once quantum information has entered cybernetics, the qubit Hilbert space and its "parent", the separable complex Hilbert space of quantum mechanics turn out to be introduced immediately as the basic mathematical formalism. Then, the "free will theorems" (Conway, Kochen 2006; 2009 ) inferable from a few properties of that Hilbert space and Minkowski space ${ }^{65}$ (due to special relativity) are inherited in the generalized, quantum-information cybernetics even extremely needing them. Indeed, a "subjective intention" was available in the original Wiener cybernetics as that of the creator of any technical system or as the pursuit of survival and reproduction of any biological system by itself. That "intention" directs the choices and information of the system at issue, and "free will" is a necessary condition for them. However, the subjects of both physics and mathematics in classical science were deprived of free will reserved for reason and human beings exceptionally. Fortunately, the theorems at issue broke that prejudice as soon as the qubit Hilbert space was relevant to whatever subject of investigation.

\section{NATURAL CYBERNETICS OF TIME}

Time orders anything. The result of ordering is information. One can introduce quantum information not only as a generalization of information relevant to infinite sets or series, but also as what time orders. In other words, time transforms quantum information into (classical) information $^{66}$. These statements can be tracked still to the foundations of mathematics being embedded in the equivalence of the axiom of choice and the well-ordering "theorem".

And vice versa as well: if one need investigate whatever as a process in time (i.e. as the gradual ordering), the mathematical formalism of the qubit Hilbert space is relevant since it is the mathematical generalization and foundation representing all the class of any processes in time as gradual ordering, i.e. as the change of information.

transmitting function of the system as a "black box" and it after interrupting feedback. One even can define the quantity of "inertia" of the system, depending on the function of negative feedback resulting into the corresponding decrease of the magnitude of transmission coefficient ("gear ratio") or function. As far as "mass" in physics is understood as corresponding to that quantity of inertia, the phenomena of "mass at rest" can be explained as a spontaneous violation of symmetry between transmissions "forwards" and "backwards' (analogically to the Higgs mechanism of the Standard model): a threshold due to a negative feedback at rest (i.e. without any output signal) and under which the output signal is zero.

${ }^{65}$ The addition of Minkowski space is redundant in a sense since the qubit Hilbert space and Minkowski space can be considered as isomorphic (Penchev 2020 June 21) and thus the conditions in terms of special relativity and Minkowski space can be rewritten thoroughly as a few more conditions inferable from the qubit Hilbert space.

${ }^{66}$ The Schrödinger equation can be interpreted so as well: it equates the change of quantum information (wave function) in time to the change of the gradient of quantum information in space to the nearest function representing a potential field. That potential field means the external physical conditions, in which the investigated quantum information exists, and thus it can be neglected as to the sense of equation (only the phrase in italic). The change of quantum information in time means classical information after the ordering by time: that is a series of qubits. The change of the gradient of quantum information in space, on the other side of the equation, represents all qubits in a coherent state without any ordering as if synchronically or simultaneously. Thus equating the two sides, (the change of) the readings of the apparatus (meant by the change of quantum information in time) and (the change of) quantum information by itself i.e. in coherent state (meant by the change of the gradient of quantum information in space) are postulated to be equal. In other words, the sense of the Schrödinger equation is: quantum information transformed into classical information after ordering in time (i.e. after measurement) and registered by the apparatus is equivalent to the same quantum information by itself (that is: before measurement). 
The methods of infinitesimal calculation in the dawn of modern physics and exact sciences during Newton's epoch meant the same (as the qubit Hilbert space): the relevant quantitative study of temporal processes, but by different mathematical tools: derivatives and differential equations $^{67}$.

Analogically, "natural cybernetics of time" can be considered as a contemporary and more powerful methodology and methods for the mathematical representation of processes in time. It supplies the corresponding process with a wave function or its partial and finite approximation (e.g. such as a neural network) equivalent to quantum information and qubits rather than with time derivatives as classical physics. That mathematical tool is suitable to investigate the way for time to appear in the researched process rather than to compare how different temporal processes run after infinitesimal calculation.

Just as involving derivatives, the introduction of quantum information (respectively, the qubit Hilbert space) is a universal approach applicable to different scientific areas rather than only to physical ones as its ancestor could do.

So what "natural cybernetics of time" means practically is the switch viewpoint ("Gestalt") from a certain physical theory (quantum mechanics or the Standard model) and its fundamental mathematical formalism (the separable complex Hilbert space) to a general methodology and methods applicable to various scientific regions and able to generate a class of relevant theories therefore sharing "quantum-information calculus" just as many classical physical theories shared the classical infinitesimal one.

Unfortunately, the prejudice that the qubit Hilbert space suits only quantum mechanics is too strong, preventing it from being multiplied into any relevant scientific domain. The cause is the misunderstanding of quantum mechanics ostensibly as a very special physical theory describing phenomena quite different from all the rest physical and consequently irrelevant, and even being ridiculous to them.

In fact, a unique circumstance, the Planck constant, indeed relevant to the microscopic scale of quantum mechanics, forced it to elaborate and develop the general "natural cybernetics of time" as a relevant mathematical tool to resolve its specific problems. Quantum mechanics needed revolutionary new methodology and methods unlike for example general relativity, another revolutionary theory but only borrowing the apparatus of infinitesimal calculus from classical physics. Its fundamental conceptual innovation was "curvature" or "curved vector space" (already non-convex after special relativity) such as pseudo-Riemannian space, i.e. all class of quantities originating from the mismatch of covariant and contravariant spaces it each point of it. Thus, the magnitude of change creating a new theory of gravitation (rather than a class of general methods absolutely necessary to be investigated a fundamental new scope and scale of

\footnotetext{
${ }^{67}$ In fact, the same tools of derivatives and differential equations are utilized in the Schrödinger equation as well. The fundamental difference is the following. The differential equations of classical physics compared different orderings in time, which was predestined to be universal as well as the absolute and unconditional premise of all claiming to be physical. Thus, the process of ordering accomplished by time therefore processing information in the final analysis was inaccessible being forbidden for science fundamentally and thoroughly. On the contrary, one involves the qubit Hilbert space to enter the intimate mechanism of ordering in-time comparing ordered and coherent (i.e. unordered and even non-orderable fundamentally) states rather than classical physics and science comparing only different orderings once time has been postulated to be sine-qua-non.
} 
human cognition as quantum mechanics $\operatorname{did}^{68}$ ) was yet surmountable by a single person though a genius.

On the contrary, quantum mechanics needed decades of geniuses in order to be established as an objective and experimental science since it had to break the scientific tradition by a revolution due to the fundamentally new kind of "non-transparent measurement" implying "God's dice" (by Einstein's famous metaphor). The resistance to the novel paradigm was so strong and relentless (in fact, continuing even today), that it was restricted forcibly only to quantum mechanics therefore prohibiting and excluding it to be extended and generalized to a methodology.

However natural cybernetics of time means the following: methods and formalisms of quantum mechanics to be applied in many other scientific areas and even generalized philosophically to temporality at all.

So, "natural cybernetics of time" can be understood as both heuristics and a method to a generalized investigation of temporality meaning its emergence after any entity being non-temporal by itself. It generalizes the classical infinitesimal calculation very fruitful to various physical processes, to processing quantum information (e.g. by the qubit Hilbert space) in order to investigate how the temporality at issue appears. Thus, it remains in the framework of science. However, it can be postulated furthermore as an unfalsifiable philosophical principle: for example, by means by "Being" and "Time" borrowed from Heidegger:

One means a projection of "Being" on "Time" to be defined "Entity" as their relation, but not necessary in general, therefore admitting that the existence of non-temporal entities or "Entity" out of "Time" can be interpreted as belonging to "Being" by itself. In other words, "natural cybernetics of time" can be interpreted properly philosophically: as an unfalsifiable doctrine claiming the asymmetry of "Being" and "Time" and the superiority of the former as the most general and fundamental philosophical category.

${ }^{68}$ In fact, the conceptual change of general relativity is commensurable with that of quantum mechanics if general relativity is granted as a generalized form of quantum gravity on virtue of the unification of smoothness (continuity) and discreteness as above. This allows for a relevant re-description of the way how temporality appears from terms of the qubit Hilbert space into those of pseudo-Riemannian space. That transition and paraphrasing is very instructive in relation to the realization of the implicit method of classical physics and science to mean the emergence of temporality by differential equations therefore involving the "relativity" of two (at least) or more changes in time. That binocularity (as the most economical, but sufficient way of multi-ocularity) to time is established definitively in pseudo-Riemannian space where one can distinguish covariant temporality from contravariant temporality changing from each other independently and resulting into the variable of curvature in any point of the space (in fact, many curvatures, or in other words, one or more tensors nonzero in general in each point of pseudo-Riemannian space). Thus, the approach of general relativity can be interpreted even as more general than that of quantum mechanics to the emergence of temporality. Quantum mechanics means the choice of a certain temporality (by measurement of the coherent state at issue) among the class of all possible ones (implicitly available in the coherent state) as the involved here concept of the "emergence of temporality". However, the concept of general relativity (or "general covariance" also coined by Einstein) can work not worse as it: the principle implies an analogical (or maybe even equivalent as the present and other papers of mine state) choice of a certain reference frame being in relative motion to the observer's reference frame. So, there is the choice of a certain temporality in both cases: however determined additionally in a different way in each case. That is: a choice of an element among its class as to quantum mechanics (and the novel notion of an "external or discrete reference frame" is relevant to the conceptual foundation of general relativity); or the same element chosen to another element of the same class and called "the observer's reference frame" as to general relativity. One can postulate that the choice is the same if both chosen element and set of alternatives are the same though described differently. 
XV INSTEAD OF CONCLUSION: NEUROSCIENCE AND THE APPLICABILITY OF NATURAL CYBERNETICS OF TIME

The "classical" Wiener cybernetics is rather outdated nowadays. It assisted the emergence of new sciences or interdisciplinary fields of research such as systemology, computer science, cognitive science, neuroscience, etc. Especially the last one is very relevant to the idea of "natural cybernetics of time" promoted here and to which it can be considered as foundation both physical and mathematical, and even philosophical.

Neuroscience postulates neural networks as a universal class of models relevant to any branch of cognition whether scientific or not: whatever be studied, a relevant subclass of models based on neural networks would exist. Indeed, the corresponding subject needs to be represented in the human brain as a relevant neural network, which links different natural entities presumably in similar ways. However, those different entities connected by the parent neural networks can be considered as again neural subnetworks situated on lower hierarchical levels. Thus unfolding all entities of all hierarchical levels, one can achieve always a complete neural network starting from a finite tuple of elements, i.e. fundamental and initial entities only recombined differently in any case of whatever scientific or empirical subject since it is represented (or representable) necessarily in human brain.

Then, neuroscience should be able to generate models of anything being cognizable by human beings. All those neural networks are finite as far as a necessary condition is to be implementable in the human brain (consisting of a huge, but finite number of neurons connected by finite numbers of axons).

As it was discussed above, the qubit Hilbert space and the class of all possible neural networks can be related to each other as a universal and omnipresent medium, in which any real neural network can be considered as a finite and thus particular and limited actualization relevant only imperfectly to a certain investigated or represented, scientific or empiric subject.

The qubit Hilbert space can serve as a scientific justification of all human cognition whether until now or future, whether valid (i.e. not falsified yet) or rejected already as partially or as fundamentally wrong. Whatever be it, it is a finite state of that medium at issue. The qubit Hilbert state can be justified by scientific transcendentalism, a falsifiable version of the unfalsifiable philosophical transcendentalism originating from the postulate of the totality fundamentally inaccessible to our experience or experiments, but implying verifiable conclusions.

Natural cybernetics of time studies just that medium and thus it can serve as a foundation of neuroscience. In turn, neuroscience supplies various applications or implementations of natural cybernetics of time for they should be finite in experience and experiments.

One can conclude that neuroscience is the "temporal and finite twin" of natural cybernetics of time, which in turn and therefore is able to serve as a foundation of the former. However, the scope and scale of natural cybernetics of time is much more extended in comparison with those of neuroscience including for example infinite neural networks or physical and mathematical entities by themselves (i.e. regardless of their eventual representation in human brain as finite neural networks whatever they be). On the other hand, the physical universe, not less relevant to that universal medium, acquires an unexpected dimension of the widest neural network ... 


\section{References:}

Bohr, Niels \& Hendrik A Kramers, and John C Slater (1924) "The quantum theory of radiation," Philosophical Magazine and Journal of Science 47 (281): 785 - 802.

Conway, John \& Simon Kochen (2006) “The Free Will Theorem," Foundations of Physics 36 (10): $1441-1473$.

Conway, John \& Simon Kochen (2006) “The Strong Free Will Theorem," Notices of the AMS 56 (2): $226-232$.

Husserl, Edmund (1891) Philosophie der Arithmetik. Psychologische und logische untersuchungen. Halle-Saale, C.E.M. Pfeffer.

Husserl, Edmund (1900 - 1901) Logische Untersuchungen. Band 1 \& 2. Leipzig, Veit.

Husserl, Edmund; Martin Heidegger (2011) Psychological and transcendental phenomenology and the confrontation with Heidegger, 1927-1931. Dordrecht - London, Springer.

Kochen, Simon \& Ernst P Specker (1967) "The problem of hidden variables in quantum mechanics," Journal of Mathematics and Mechanics 17 (1): 59 - 87.

Lewin, Kurt (1936) Principles of topological psychology (translated by Fritz Heider and Grace M. Heider). New York, McGraw-Hill Book Company.

Von Neumann, John (1932) Mathematische Grundlagen der Quantenmechanik. Berlin, J Springer.

Penchev, Vasil (2020 July 20) "The Relationship of Arithmetic as Two Twin Peano Arithmetic(s) and Set Theory: A New Glance from the Theory of Information," SSRN: https://ssrn.com/abstract=3656179 or http://dx.doi.org/10.2139/ssrn.3656179.

Penchev, Vasil (2020 July 9) 'A Class of Examples Demonstrating that 'P $\neq$ NP' in the 'P vs NP' Problem," SSRN: https://ssrn.com/abstract=3647038 or http://dx.doi.org/10.2139/ssrn.3647038 .

Penchev, Vasil (2020 October 18) "Natural Cybernetics and Mathematical History: The Principle of Least Choice in History," SSRN: https://ssrn.com/abstract=3714119 or http://dx.doi.org/10.2139/ssrn.3714119.

Penchev, Vasil (2020 June 18) "Quantum Information as the Information of Infinite Series," SSRN: https://ssrn.com/abstract=3630063 or http://dx.doi.org/10.2139/ssrn.3630063 .

Penchev, Vasil (2020 August 17) "Quantum-Information Conservation. The Problem about 'Hidden Variables', or the 'Conservation of Energy Conservation' in Quantum Mechanics: A Historical Lesson for

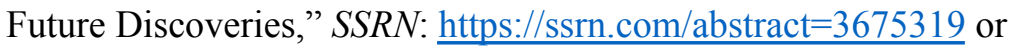
http://dx.doi.org/10.2139/ssrn.3675319.

Penchev, Vasil (2020 August 31) "Two deductions: (1) from the totality to quantum information conservation; (2) from the latter to dark matter and dark energy," SSRN: https://ssrn.com/abstract=3683658 or http://dx.doi.org/10.2139/ssrn.3683658 .

Penchev, Vasil (2020 August 30) "The Case of Quantum Mechanics Mathematizing Reality: The 'Superposition' of Mathematically Modeled and Mathematical Reality: Is There Any Room for Gravity?' SSRN: https://ssrn.com/abstract=3683342 or http://dx.doi.org/10.2139/ssrn.3683342 .

Penchev, Vasil (2020 June 21) "The Isomorphism of Minkowski Space and the Separable Complex Hilbert Space and Its Physical Interpretation," SSRN: https://ssrn.com/abstract=3632159 or http://dx.doi.org/10.2139/ssrn.3632159.

Penchev, Vasil (2019) "Why Anything Rather Than Nothing? The Answer of Quantum Mechanics," in Ivan Mladenov \& Aleksandar Feodorov (eds.), Non/Cognate Approaches: Relation \& Representation. Sofia: "Парадигма", pp. 151-172. (Available at SSRN:

https://ssrn.com/abstract $=3644936$ )

Penchev, Vasil (2010) "Insolubility of the first incompleteness theorem. Gödel and Hilbert mathematics" (in Bulgarian, original title "Неразрешимост на първата теорема за непълнотата. Гьоделова и Хилбертова математика"), Philosophical Alternatives 19 (5): 104-119. (Available at PhilPapers: https://philpapers.org/rec/PEN-21 ) 
Penchev, Vasil (2013) "The Kochen - Specker theorem in quantum mechanics: a philosophical comment" (part 1 \& part 2), Philosophical Alternatives 22 (1) \& (3): 67-77 \& 74-83. (Available at PhilPapers: https://philpapers.org/rec/PENTK-2 \& https://philpapers.org/rec/PENTK ).

Schrödinger, Erwin (1935) "Die gegenwärtige Situation in der Quantenmechanik," Naturwissenschaften 23 (48) 807-812; 23 (49), 823-828; 23 (50), 844-849.

Shannon, Claude E (1948) "A Mathematical Theory of Communication," Bell System Technical Journal 27 (3) \& (4): 379-423 \& 623-666.

Wiener, Norbert (1948) Cybernetics: Or Control and Communication in the Animal and the Machine. Cambridge (Mass.) MIT press.

Wiener, Norbert (1950) The Human use of human beings, cybernetics and society. Boston, Houghton Mifflin Co.

Wiener, Norbert (1964) God \& Golem, Inc.: A Comment on Certain Points Where Cybernetics Impinges on Religion. Cambridge (Mass.), MIT Press. 